\title{
Colliding-wind binary systems: Diffusive shock acceleration and non-thermal emission
}

\author{
J. M. Pittard ${ }^{1 \star}$, G. S. Vila ${ }^{\dagger} \dagger$ and G. E. Romero ${ }^{2}$ \\ ${ }^{1}$ School of Physics and Astronomy, University of Leeds, Woodhouse Lane, Leeds LS2 9JT, UK \\ ${ }^{2}$ Instituto Argentino de Radioastronomía, CCT-La Plata, CONICET, 1900FWA, La Plata, Argentina
}

Accepted ... Received ....; in original form ...

\begin{abstract}
We present a model for the non-thermal emission from a colliding-wind binary. Relativistic protons and electrons are assumed to be accelerated through diffusive shock acceleration (DSA) at the global shocks bounding the wind-wind collision region. The non-linear effects of the back-reaction due to the cosmic ray pressure on the particle acceleration process and the cooling of the non-thermal particles as they flow downstream from the shocks are included. We explore how the non-thermal particle distribution and the $\mathrm{keV}-\mathrm{GeV}$ emission changes with the stellar separation and the viewing angle of the system, and with the momentum ratio of the winds. We confirm earlier findings that DSA is very efficient when magnetic field amplification is not included, leading to significantly modified shocks. We also find that the non-thermal flux scales with the binary separation in a complicated way and that the anisotropic inverse Compton emission shows only a moderate variation with viewing angle due to the spatial extent of the wind-wind collision.
\end{abstract}

Key words: binaries: general - gamma-rays: stars - radiation mechanisms: nonthermal - stars: early-type - stars: winds, outflows - stars: Wolf-Rayet

\section{INTRODUCTION}

Colliding-wind binary (CWB) systems consist of two earlytype stars with powerful winds (e.g., Stevens, Blondin \& Pollock 1992; Pittard 2009). If the strength of the winds is not too unbalanced, and/or if the stars are widely separated, the winds will collide at supersonic speeds between the stars. This produces a wind-wind collision region (WCR) where strong global shocks slow the winds and heat the plasma up to temperatures of $10^{7} \mathrm{~K}$ or more.

In some systems the global shocks are collisionless, and are mediated by magnetic fields rather than through coulombic particle interactions. This allows particles to undergo diffusive shock acceleration (DSA), such that a small fraction obtain relativisitic energies (e.g., Eichler \& Usov 1993; Benaglia \& Romero 2003; Dougherty et al. 2003; Reimer, Pohl \& Reimer 2006; Pittard et al. 2006; Pittard \& Dougherty 2006). The presence of such particles has been confirmed via radio observations which display a negative spectral index for the flux density $\left(S_{v} \propto v^{\alpha}\right.$, with $\left.\alpha<0.0\right)$, and which is interpreted as synchrotron radiation. In some systems the non-thermal emission is spatially resolved and is located at

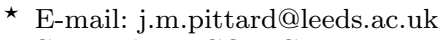

$\dagger$ Currently at CONICET in YPF Tecnología S.A. the assumed position of the WCR (e.g., Williams et al. 1997; Dougherty, Williams \& Pollacco 2000; Dougherty et al. 2005; O'Connor et al. 2005; Dougherty \& Pittard 2006; Ortiz-León et al. 2011; Benaglia et al. 2015; Brookes 2016). In other systems the non-thermal radio emission is not spatially resolved but is linked to orbital variability (e.g., Blomme et al. 2013, 2017).

In contrast to the situation in the radio band, confirmation of non-thermal X-ray and $\gamma$-ray emission from CWBs has proved extremely challenging. Until last year the best evidence was a Fermi source located near to $\eta$ Carinae (e.g., Reitberger et al. 2015), an extreme and unusual CWB composed of an LBV primary in orbit with an as yet unobserved companion which also has a fast and powerful wind (e.g., Pittard \& Corcoran 2002; Corcoran 2005; Hamaguchi et al. 2007; Damineli et al. 2008; Okazaki et al. 2008; Parkin et al. 2009; Corcoran et al. 2010; Mehner et al. 2010; Parkin et al. 2011; Madura et al. 2013). A second Fermi source is associated with $\gamma^{2}$ Velorum, while upper limits exist for several other WR+O star CWBs (Pshirkov 2016). However, the angular resolution of the Fermi telescope is relatively poor, and the source circles are large. Thus it remained possible that the $\gamma$-ray emission detected by Fermi may actually be coming from other sources than the CWBs (Benaglia 2016).

This situation dramatically changed last year when non- 
thermal X-ray emission from $\eta$ Carinae was detected with NuSTAR, a focusing telescope (Hamaguchi et al. 2018). These observations narrowed down the position of the nonthermal emission to within several arc-seconds of the star, and showed that it varied with the orbital phase of the binary. In addition, the photon index of the non-thermal Xray emission was similar to that found for the $\gamma$-ray spectrum. This is the conclusive proof that has long been sought, and the NuSTAR observations provide the crucial support that the detections at X-ray energies (Leyder et al. 2008; Sekiguchi et al. 2009; Leyder et al. 2010; Hamaguchi et al. 2014), GeV energies (Tavani et al. 2009; Abdo et al. 2010; Farnier et al. 2011; Reitberger et al. 2012, 2015; Balbo \& Walter 2017) required. The latest development is the detection of $\eta$ Carinae at energies of 100 's GeV by the HESS telescope (H.E.S.S. collaboration 2020).

In this paper we develop a model for the relativistic particles in CWBs and the resulting high-energy nonthermal emission (for previous models see, e.g., Dougherty et al. 2003; Pittard et al. 2006; Pittard \& Dougherty 2006; Reimer et al. 2006; Bednarek \& Pabich 2011; Reitberger et al. 2014a,b; Ohm et al. 2015; del Palacio et al. 2016; Reitberger et al. 2017; Grimaldo et al. 2019). Our model is similar to that of del Palacio et al. (2016) but differs in several ways. The most significant difference is that we use the semi-analytic model of Blasi, Gabici \& Vannoni (2005) to calculate the post-shock non-thermal particle distribution. We confirm earlier findings that DSA is very efficient when magnetic field amplification is not included (e.g. Grimaldo et al. 2019). Our current focus is the non-thermal X-ray and $\gamma$-ray emission that extends up to $10 \mathrm{GeV}$. In Sec. 2 we describe our new model. In Sec. 3 we present the results and we summarize and conclude in Sec. 4.

\section{THE MODEL}

To better predict and understand the non-thermal emission from CWBs we have developed a new, fast and efficient, numerical model. While models based on hydrodynamical simulations are best able to capture complex behaviour such as the curvature and skew of the WCR resulting from orbital dynamics, or the nature of the flow within the WCR, they are more cumbersome and costly to calculate (especially in 3D). Therefore, there is a place for simpler and faster calculations that are based on an analytic description of the position of the contact discontinuity (CD) between the shocked stellar winds. In the following subsections we describe the geometry of our model, the acceleration and subsequent cooling of the non-thermal particles in it, and the non-thermal emission processes that are included in our calculations. We conclude this section with details about our "standard model".

\section{$2.1 \quad$ The geometry}

Our model is based on an axisymmetric description of the WCR in which it is assumed that the winds collide at constant speeds (we take this to be the terminal speeds of the winds). Thus, orbital effects and the acceleration/deceleration of the winds are ignored. Our models are therefore most appropriate for wide binaries with long orbital periods where these neglected effects are minimised ${ }^{1}$. We also assume that the global shocks are coincident with the CD. This is not true in systems where the cooling length of the shocked plasma is comparable to the stellar separation (or "size" of the WCR), since the shocks stand-off from the CD in such cases (see, e.g., Pittard \& Dawson 2018). However, it provides a useful first order approximation for the shock positions.

The position of the CD is computed using the equations in Cantó et al. (1996). From the apex of the WCR the CD is divided into segments of 1 degree intervals measured from the secondary star (hereafter assumed to be the star with the less powerful wind). At the centre point of each segment the pre-shock wind properties are calculated: the density, $\rho_{0}$, and the velocity parallel $\left(u_{0 \|}\right)$ and perpendicular $\left(u_{0 \perp}\right)$ to the CD.

Each shock segment has two coincident streamlines that flow downstream along the $\mathrm{CD}$, one for the non-thermal electrons and one for the non-thermal protons. Each streamline is split into zones. The size/depth of these zones is controlled by the requirement that the highest energy particles lose less than 10 per cent of their energy in any one step (this is why we use two streamlines: the high-energy non-thermal electrons cool very quickly, which requires small zones, while the non-thermal protons cool much more slowly and larger zones can be used). This ensures that the cooling is properly resolved. There may be many zones per segment. We follow the post-shock non-thermal particles for a distance of $10 D$ downstream of their acceleration point, where $D$ is the stellar separation.

As the particles flow along the streamline they move from the centre of the current segment towards its edge at a speed of $u_{0 \|}$. If the particles are about to move into the next segment the timestep is adjusted so that they only just cross into it. When they cross into the next segment the target photon flux and post-shock particle density and magnetic field of the new segment replace the corresponding values from the older segment. In this way there is a reduction in the rate that the particles cool via inverse Compton, synchrotron, coulombic and proton-proton cooling, reflecting the reduction in target photon flux and particle densities along the CD. The velocity of the flow along the streamline is also updated when the streamline moves into the next segment, so that the particles gradually accelerate along their streamline.

For the purpose of calculating the emission we gather the particles in each zone to the centre of the segment that the zone is in. We then create azimuthal patches by rotating the $\mathrm{CD}$ around the line-of-centres. For the work presented in this paper we create 8 azimuthal patches per CD segment.

\subsection{The diffusive shock acceleration}

The main difference to del Palacio et al. (2016)'s work concerns the calculation of the non-thermal particle spectrum at each global shock. del Palacio et al. (2016) assume that the non-thermal particles at the two stellar-wind shocks have an energy distribution at injection of $Q(E) \propto E^{-p}$. The initial

1 Note that Parkin \& Pittard (2008) developed a simple model for the WCR that did approximate orbital effects. 
post-shock distribution at each position along each shock is then given by $N_{0}(E)=Q(E) t_{\mathrm{adv}}$, where $t_{\mathrm{adv}}$ is the time for the particles to be advected downstream into the next cell. The distribution is normalized by the local fraction of the incoming kinetic energy flux perpendicular to the shock surface that is converted into non-thermal particles, $f_{\mathrm{NT}}$.

In contrast, we solve the diffusion-convection equation for the cosmic rays using the semi-analytic model of Blasi et al. (2005) to obtain the immediate post-shock particle distribution at each shock-segment. The diffusion of the nonthermal particles is assumed to be energy dependent in this model (specifically, it is an increasing function of energy), and is close to Böhm-like (see Fig. 5 in Blasi et al. 2005). This means that the spectral index of the particle distribution, $p$, can also be energy dependent due to the shock modification process that occurs when DSA is efficient. This is a major difference to the del Palacio et al. (2016) model where the non-thermal particles are assumed to exert no back-reaction on the thermal plasma.

The Blasi et al. (2005) shock acceleration model depends on a number of parameters, such as the pre-shock velocity and Mach number of the flow normal to the shock $\left(u_{0 \perp}\right.$ and $\left.M_{0 \perp}\right)$, and the maximum and injected momenta of the particles $\left(p_{\max }\right.$ and $\left.p_{\text {inj }}\right)$. The latter is set through the parameter $\chi_{\text {inj }}=p_{\text {inj }} / p_{\text {th }}$ where $p_{\text {th }}$ is the momentum of particles in the thermal peak of the Maxwellian distribution in the downstream plasma. $M_{0 \perp}$ depends on the pre-shock gas temperature which we set to $T_{0}=10^{4} \mathrm{~K}$ as appropriate for photoionized stellar winds. $\chi_{\mathrm{inj}}$ is a free parameter in Blasi et al. (2005)'s model but, as suggested, we use a default value of $\chi_{\mathrm{inj}}=3.5$.

Blasi et al. (2005)'s model assumes that the shock is parallel, which means that the magnetic field is not amplified across the shock. Nor does the magnetic field become dynamically important. In reality, the orientation of the preshock magnetic field will vary in a complicated fashion over the WCR. For wide binaries, and where the spin-axis of each star aligns with the orbital axis, perpendicular shocks are expected at the apex of the WCR, but other regions of the WCR may have quasi-parallel shocks. In addition, if the cosmic rays strongly amplify the magnetic field the far upstream orientation may become almost irrelevant. These complications are beyond the current work: instead, we do not worry about shock obliquity except to determine its effect on the pre-shock and post-shock velocities normal to the shock, and we treat the shocks as being parallel along their entire surfaces. The magnetic field strength in CWBs is also typically very uncertain, so we treat the pre-shock magnetic field as a free-parameter in the model: we set its strength through the parameter $\zeta_{\mathrm{B}}=U_{\mathrm{B}} / U_{\mathrm{KE}}$, where $U_{\mathrm{B}}$ and $U_{\mathrm{KE}}=0.5 \rho_{0} v_{\infty}^{2}$ are the pre-shock magnetic and kinetic energy densities, respectively, and we require that $\zeta_{\mathrm{B}}<1$. The pre-shock magnetic flux density, $B_{0}$, is then given by $B_{0}=\sqrt{8 \pi U_{\mathrm{B}}}$. Finally, we assume that the magnetic field is not compressed across the shock, consistent with our use of Blasi et al. (2005)'s model and the assumption that the shock is parallel. In future work we will consider magnetic field amplification in our model.

Although Blasi et al. (2005)'s model is for parallel shocks, it does depend implicitly on the pre-shock magnetic field, which affects the value of $p_{\max }$. This is because the maximum momentum of the non-thermal particles, $p_{\max }$, is set by the diffusion (escape) of particles from the shock, where the diffusion length $l_{\text {diff }}=r_{\text {shk }} / 4$, and where $r_{\text {shk }}$ is the distance of the shock from the star. This gives a maximum proton energy $E_{\max }=l_{\text {diff }} e B_{0} u_{0 \perp} / c$. An exponential cut-off is then applied to the non-thermal proton spectrum at $p_{\max }$.

The non-thermal electron spectrum has its own maximum momentum, $p_{\text {max,e }}$, which is calculated by balancing the local acceleration and loss rates, and is similarly truncated at high energies. Due to the strong inverse Compton cooling in these systems $p_{\max , \mathrm{e}}<<p_{\max }$. The non-thermal electron spectrum is normalized to the non-thermal proton spectrum by setting $f_{\mathrm{pe}}=e p_{\text {ratio }} f_{\mathrm{pp}}$, where $f_{\mathrm{pe}}$ and $f_{\mathrm{pp}}$ are the electron and proton particle distributions and $e p_{\text {ratio }}$ is the ratio of the electron to proton number density at high energies. The particle distributions are typically calculated for 140 logarithmic bins in momentum space from $10^{-6}-10^{8} m_{\mathrm{p}} c$.

\subsection{The kinetic equation}

The non-thermal particle spectrum downstream of the shock is calculated by solving the kinetic equation. For a volume co-moving with the underlying thermal gas, and ignoring diffusion, particle injection and escape, the energy distribution $n \equiv d N / d E$ as a function of time $t$ and energy $E$ is given by the continuity equation (Ginzburg \& Syrovatskii 1964; Blumenthal \& Gould 1970)

$\frac{\partial n(E, t)}{\partial t}+\frac{\partial(\dot{E} n(E, t))}{\partial E}=0$.

The second term is an advection term in energy space due to cooling processes (e.g. synchrotron, relativistic bremsstrahlung, inverse Compton - see next subsection). This equation is valid when the energy losses can be treated as continuous: if the particles lose a large fraction of their total energy in a single collision then the exact integrodifferential equation must instead be used. However, significant differences in the electron distributions only occur if both the electron injection function and the ambient radiation field are mono-energetic (Zdziarski 1989). If the target photon field is a black-body the continuous energy losss approximation differs by less than 20 per cent from the exact solution for electron energies $<10 \mathrm{TeV}$ (Khangulyan \& Aharonian 2005). This difference will be further reduced due to the increasing importance of the continuous synchrotron energy losses for the highest energy particles. A continuous treatment for the hadronic interactions is justified by the large number of inelastic collisions that allow one to move from the summation to an integral (Stecker 1971).

If we define the quantity $\tau\left(E, E^{\prime}\right)$ as the time required to cool from an energy $E^{\prime}$ to $E\left(\leq E^{\prime}\right)$,

$\tau\left(E, E^{\prime}\right)=\int_{E}^{E^{\prime}} \frac{d E^{\prime \prime}}{\dot{E}\left(E^{\prime \prime}\right)}$

then the evolved distribution at time $t$ of the immediate postshock distribution $n(E, 0)$ is

$n(E, t)=\frac{\dot{E}\left(E^{\prime}\right)}{\dot{E}(E)} n\left(E^{\prime}, 0\right)$,

where $E^{\prime}$ satisfies $\tau\left(E, E^{\prime}\right)=t$ (cf. del Palacio et al. 2016). For a given $E$ and $t$ we determine $E^{\prime}$ using a standard numerical root-finding technique. As noted earlier, Eq. 3 is evaluated along the post-shock streamline at the shorter of the two time intervals for either the maximum energy of the particles 
to decrease by 10 per cent or for the flow to move into the next segment along the CD.

\subsection{Cooling of the downstream non-thermal particles}

Post-shock energy losses for the non-thermal electrons occur because of inverse Compton emission, synchrotron emission, relativistic bremsstrahlung, coulombic cooling and adiabatic cooling. We assume that the energy loss is continuous and occurs in a fully ionized plasma. Further details of the cooling are noted in App. A.

For the primary electrons, synchrotron cooling is always sub-dominant to inverse Compton cooling in our models (in the Thomson regime their relative strength scales as $\left.U_{\mathrm{B}} / U_{\mathrm{ph}}\right)$. The effect of adiabatic cooling on the non-thermal electron distribution is usually seen most strongly at relatively low energies (the high energy electrons cool rapidly through inverse Compton cooling before they have had the opportunity to flow very far downstream - see Figs. 6 and 7).

The non-thermal proton distribution is also subject to cooling as it flows downstream of the shocks, and in this work we consider proton-proton pion production and adiabatic expansion, and again assume that the energy loss is continuous. Since we only consider acceleration of nonthermal protons in this work, we do not need to consider the fragmentation of non-thermal nuclei (e.g. He nuclei) due to collisions with either thermal ions or photons (the latter being photodisintegration).

\subsection{Non-thermal emission processes}

For a specified upscattered photon energy we obtain the anisotropic IC photon flux from each azimuthal patch by integrating over the non-thermal particle distribution, the incident black-body photon distribution from each star, and the azimuthal and polar angles of points on each stellar surface. The anisotropic inverse Compton emission calculation follows Cerutti (2007) and Vila (2012) ${ }^{2}$, and some details are noted in App. B1. Three rotations of the coordinate system are used to convert a given line of sight into the coordinate frame used in Fig. B2.

The relativistic bremsstrahlung emission from nonthermal electrons colliding with thermal protons is calculated using the prescription noted in App. B2. The $\gamma$-ray emission from the decay of neutral pions produced in collisions between thermal and non-thermal protons is calculated in the delta functional approximation using the prescription noted in App. B3.

\subsection{Neglected processes}

Our focus in this paper is the non-thermal X-ray and $\gamma$-ray emission up to $10 \mathrm{GeV}$. As a result we do not calculate the synchrotron emission, which we do not expect to exceed energies of $\sim 10^{3}-10^{4} \mathrm{eV}$ (see Eq. 4). We also do not include

2 These theses are available at http://citeseerx.ist.psu.edu/ viewdoc/download?doi=10.1.1.1019.4046\&rep=rep1\&type=pdf and at https://inis.iaea.org/search/search.aspx?orig_q= $\mathrm{RN}: 46026940$. photon-photon absorption (which is inefficient below incident photon energies of $\sim 10-100 \mathrm{GeV}$ ), or consider emission from the thermal particles. Finally, we do not consider the formation of, and emission from, secondary particles. Each of these processes will be examined in future work.

\section{7 $\quad$ Standard parameters}

In keeping with previous studies (Dougherty et al. 2003; Pittard et al. 2006) we examine the emission from a "standard" CWB model of a WR+O system with the parameter values as noted in Table 1. We refer to the WR star as the "primary" and the O star as the "secondary". The wind momentum ratio, $\eta=\dot{M}_{\mathrm{O}} v_{\infty, \mathrm{O}} / \dot{M}_{\mathrm{WR}} v_{\infty, \mathrm{WR}}=0.1$, and the distance of the stagnation point from the $\mathrm{WR}$ and $\mathrm{O}$ star is respectively $r_{\mathrm{WR}}=0.74 \mathrm{D}$ and $r_{\mathrm{O}}=0.26 \mathrm{D}$. Fig. 1 displays the structure of the CD corresponding to $\eta=0.1$ as in our standard system.

With such parameters the WCR is largely adiabatic, which means that the global shocks that decelerate each wind stand-off from the CD by some significant distance. However, we repeat that for the purposes of this work we assume that the global shocks and the CD are coincident. We also assume that the winds are composed of pure hydrogen for the DSA model, but temperatures are calculated assuming that the average particle mass for both winds is $\mu=0.6 m_{\mathrm{H}}$ (i.e. solar abundances). Pre-shock wind temperatures of $10^{4} \mathrm{~K}$ are assumed. The WR star is located at $(z, r)=(0,0)$ while the O-star is at $(z, r)=(D, 0)$. We assume that $\zeta_{\mathrm{B}}=10^{-3}$ and $\chi_{\mathrm{inj}}=3.5$.

The pre-shock density and Mach number of both winds at the stagnation point are $\rho_{0}=2.2 \times 10^{-19} \mathrm{~g} \mathrm{~cm}^{-3}$ and $M_{0 \perp}=132$. The pre-shock kinetic energy density, $U_{\mathrm{KE}}=$ $0.5 \rho_{0} v^{2}=4.4 \times 10^{-3} \mathrm{erg} \mathrm{cm}^{-3}$. The pre-shock magnetic energy density, $U_{\mathrm{B}}=\zeta_{\mathrm{B}} U_{\mathrm{KE}}=4.4 \times 10^{-6} \mathrm{erg} \mathrm{cm}^{-3}$, giving $B_{0}=0.01 \mathrm{G}$. The total photon energy density (from both stars) at the stagnation point is $U_{\mathrm{ph}}=0.021 \mathrm{erg} \mathrm{cm}^{-3}$. The maximum proton momenta at the WR-star shock and at the O-star shock are $p_{\text {max }}=8.5 \times 10^{3} \mathrm{~m}_{\mathrm{p}} \mathrm{c}$ and $2.7 \times 10^{3} \mathrm{~m}_{\mathrm{p}} \mathrm{c}$, respectively. The WR-shock accelerates particles up to higher energies because the incoming wind has a greater radius of divergence (i.e. it is more planar) than the O-star wind impinging on the $\mathrm{O}$ star shock.

The maximum electron Lorentz factor from each shock is $\gamma_{\text {max }, \mathrm{e}} \approx 5 \times 10^{6}\left(p_{\text {max,e }} \approx 2700 \mathrm{~m}_{\mathrm{p}} \mathrm{c}\right)$, and is slightly higher for the WR-star shock than for the O-star shock. In the former case it is limited by inverse Compton cooling, while in the latter case it is limited by the maximum energy of the protons. The treatment of $p_{\max }$ and $p_{\max , \mathrm{e}}$ in the current work is significantly different compared to our previous work where it was assumed that $p_{\max }=p_{\text {max,e }}$, and that these values were the same for both shocks and along each shock (Dougherty et al. 2003; Pittard et al. 2006; Pittard \& Dougherty 2006). In this sense our new calculations are more realistic.

For our standard parameters, and with the assumption that the post-shock magnetic field strength is equal to the pre-shock value, we find that synchrotron cooling is always sub-dominant to inverse Compton cooling for the primary electrons - see Fig. 6. However, synchrotron cooling could become dominant in situations where the magnetic field is compressed. This will be examined further in future work. 


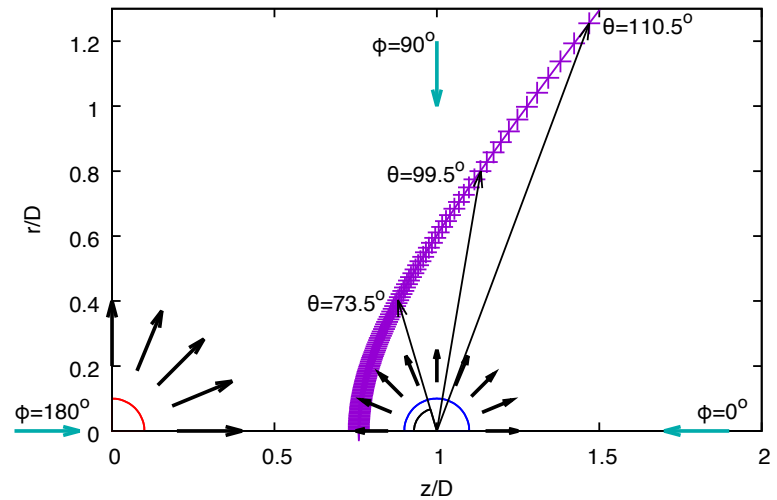

Figure 1. The position of the contact discontinuity (CD) in our standard model. The primary star is indicated by the red circle, and the secondary star by the blue circle. Note that the stars are not drawn to scale. The stagnation point of the WCR is at $(z, r)$ $=(0.74,0.0) D . \theta$ is the angle between the line of centres between the primary and secondary stars and a position on the CD, as measured from the secondary star. Marks along the CD indicate the centre of segments of $d \theta=1^{\circ}$ width as seen from the secondary star. The mark that is furthest downstream corresponds to the $111^{\text {th }}$ segment $\left(\theta=110.5^{\circ}\right.$ at its centre). The viewing angle $\phi$ indicates the angle of the line of sight to the observer. The secondary star is in front when $\phi=0^{\circ}$, the system is at quadrature when $\phi=90^{\circ}$, and the primary star is in front when $\phi=180^{\circ}$. For most of our calculations we adopt $\phi=90^{\circ}$. For the purpose of our model we assume that the global shocks which decelerate each wind are coincident with the CD.

Table 1. The stellar parameters used in our standard model. Both stars are assumed to have an effective temperature $T=$ $40,000 \mathrm{~K}$. The stellar separation, $D=2 \times 10^{15} \mathrm{~cm}$.

\begin{tabular}{lll}
\hline Parameter & WR star & O star \\
\hline$\dot{M}\left(\mathrm{M}_{\odot} \mathrm{yr}^{-1}\right)$ & $2 \times 10^{-5}$ & $2 \times 10^{-6}$ \\
$v_{\infty}\left(\mathrm{km} \mathrm{s}^{-1}\right)$ & 2000 & 2000 \\
$L\left(\mathrm{~L}_{\odot}\right)$ & $2 \times 10^{5}$ & $5 \times 10^{5}$ \\
\hline
\end{tabular}

Synchrotron emission also occurs mostly below the energy range that is of interest to the current work. The synchrotron emission from a single non-thermal electron cuts off at energies above

$E=\frac{3 h \gamma_{\mathrm{e}}^{2} e B \sin \alpha}{4 \pi m_{\mathrm{e}} c}$.

With $\gamma_{\text {max,e }}=5 \times 10^{6}$ and $\sin \alpha=1$ this gives $E \approx 4.3 \times 10^{5} B \mathrm{eV}$. The pre-shock (and post-shock) magnetic field strength in our standard model at the apex of the WR-star and O-star shocks is $B_{0}=0.01 \mathrm{G}$, which gives $E=4200 \mathrm{eV}$. In this paper we do not calculate the synchrotron emission (as our focus is on the $\mathrm{keV}-\mathrm{GeV}$ energy range).

\section{RESULTS}

We begin by examining various quantities along each shock. We then examine the distribution of non-thermal particles, and finally investigate how the predicted emission changes as various parameters are altered. Unless otherwise noted we adopt our "standard" parameters, in which the stellar separation $D=2 \times 10^{15} \mathrm{~cm}$, and the viewing angle $\phi=90^{\circ}$ (i.e. the line-of-sight is perpendicular to the line-of-centres between the stars - see Fig. 1).

\subsection{The standard model}

\subsubsection{Quantities along each shock}

Fig. 2 shows various quantities from our standard model as a function of angle, $\theta$, along the CD as measured from the secondary star $\left(\theta=0^{\circ}\right.$ corresponds to the stagnation point of the WCR on the line-of-centres between the stars, while $\theta=$ $90^{\circ}$ indicates a point on the CD where $z=D$ ). The maximum value of $\theta$ is 180 degrees minus the half-opening angle of the WCR. For our standard parameters, $\theta_{\max } \approx 130^{\circ} \cdot r, z$ and $l$, the distance along the CD from the stagnation point, increase rapidly as $\theta$ approaches its maximum value.

Fig. 2b) shows the perpendicular pre-shock WR- (solidline) and O- (dashed line) wind velocity as a function of $\theta$. At the stagnation point the winds collide head-on and $u_{0 \perp}$ is equal to the terminal wind speeds. As one moves off-axis the shocks become gradually more oblique (the WR-shock becomes more oblique more rapidly), and the perpendicular pre-shock velocity decreases, reaching zero when $\theta=\theta_{\max }$.

Fig. 2c) shows the pre-shock WR- (solid-line) and O(dashed line) wind density as a function of $\theta$. Both densities are identical at the stagnation point $\left(\rho_{0}=2.2 \times 10^{-19} \mathrm{~g} \mathrm{~cm}^{-3}\right)$ due to the fact that the winds collide at the same speed. The pre-shock WR wind density falls off more slowly with increasing $\theta$ than the pre-shock $\mathrm{O}$ wind density. Since the pre-shock wind temperatures are fixed at $10^{4} \mathrm{~K}$, the preshock wind pressures in Fig. 2d) show the same behaviour with $\theta$ as the pre-shock wind densities. Similarly, the preshock perpendicular Mach number of each wind behaves in the same way as the pre-shock perpendicular wind speeds (compare Fig. 2b and e). The on-axis pre-shock perpendicular Mach number is $M_{0 \perp}=132$.

The maximum non-thermal proton momentum at each shock is shown in Fig. 2f). $p_{\max }$ is nearly $10^{4} \mathrm{~m}_{\mathrm{p}} \mathrm{c}$ for the WR shock and declines off-axis. The value of $p_{\max }$ is about 4 times smaller for the $\mathrm{O}$ shock due to the reduced distance of the shock from the star (see Sec. 2.2).

Fig. 2 showed various pre-shock parameters, including some that are needed for the Blasi et al. (2005) DSA model. In Fig. 3 we show various outputs from Blasi et al. (2005)'s model. Fig. 3a) shows $R_{\text {tot }}$, the shock total compression ratio. Strong shocks in gas with a ratio of specific heats $\gamma=5 / 3$ have a compression ratio of 4 , but $R_{\text {tot }}$ can increase significantly when DSA efficiently accelerates non-thermal particles that then escape upstream from the shock. This is indeed the case in our standard model, where we see that $R_{\mathrm{tot}}$ reaches values of order $40^{3}$. The lower value of $p_{\max }$ on the line-of-centres for the $\mathrm{O}$ shock causes $R_{\text {tot }}$ to be slightly lower than for the WR shock. $R_{\text {tot }}$ decreases with increasing $\theta$ as the shocks become more oblique, and $u_{0 \perp}, M_{0 \perp}$ and $p_{\max }$ all decline.

3 Less extreme compression ratios are achieved when the dynamical feedback of the magnetic field amplification is considered (see, e.g., Caprioli et al. 2009). 

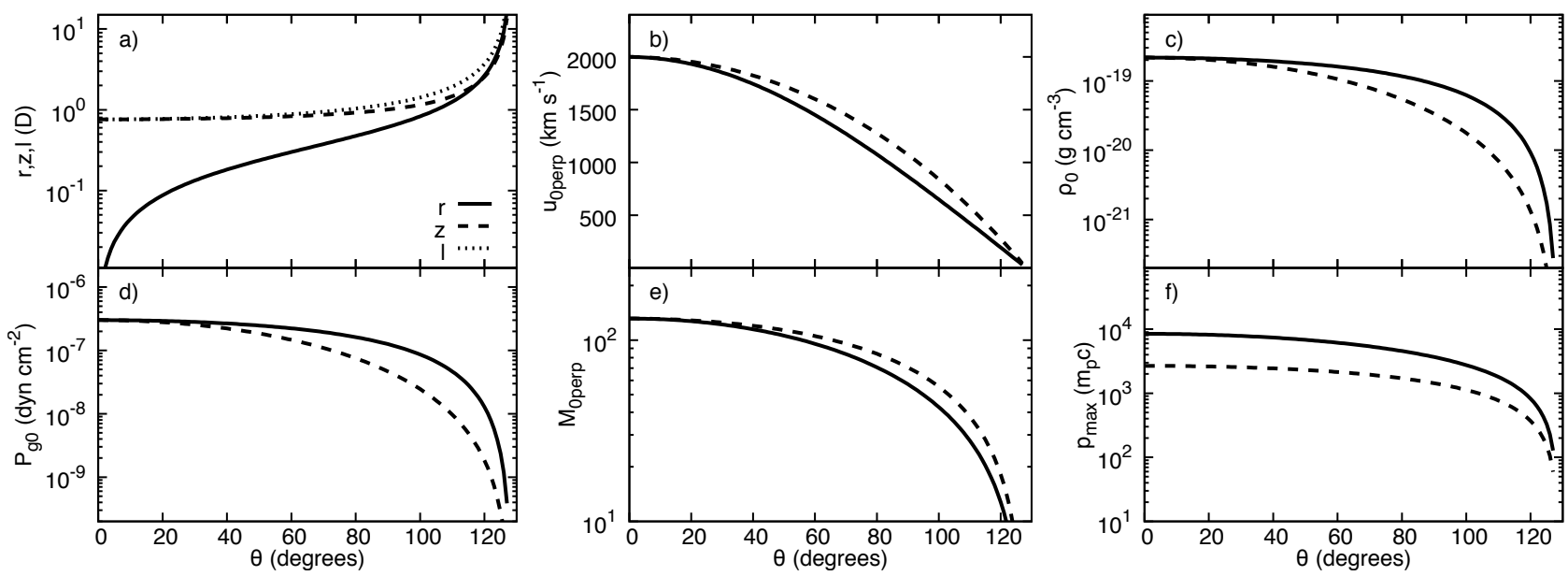

Figure 2. Selected quantities along the CD as a function of the angle $\theta$ from the secondary star. Panel a) shows the $r$ and $z$ position of the CD segment, and the distance $l$ along the CD. Panels b-e) show the pre-shock perpendicular wind velocity, density, thermal gas pressure, and perpendicular Mach number, respectively, while panel f) shows the maximum non-thermal proton momentum. In panels b-f) the solid line indicates the properties for the WR-star shock, while the dashed line indicates the properties for the O-star shock.

Fig. 3b) shows the compression ratio across the subshock, $R_{\text {sub }}$. The sub-shock is a discontinuity in the overall shock structure. $R_{\text {sub }}$ is $\approx 3.5$ for both the WR and O shock and decreases slightly as $\theta$ increases, before falling rapidly as $\theta \rightarrow \theta_{\max }$. The sub-shock, plus any shock-precursor, is responsible for heating the thermal plasma.

The post-shock thermal, $P_{\mathrm{g}}$, and non-thermal, $P_{\mathrm{c}}$, particle pressure is shown in Fig. $3 \mathrm{c}$ ). It is clear that $P_{\mathrm{c}}$ exceeds $P_{\mathrm{g}}$ by a factor of 100 at the shock apex. This difference reduces as $\theta$ increases, until at large $\theta$ the value of $P_{\mathrm{c}}$ drops to a value similar to that of $P_{\mathrm{g}}$ as DSA becomes less efficient.

In Fig. 3d) we see the variation with $\theta$ of the fraction of the incoming WR-wind kinetic energy flux that is advected downstream in non-thermal particles, $F_{\text {adv }}$. Also shown is the fraction that is carried upstream by escaping non-thermal particles, $F_{\text {esc }}$, and the total non-thermal particle flux $\left(F_{\text {tot }}=\right.$ $\left.F_{\text {adv }}+F_{\text {esc }}\right)$. Due to the efficient DSA that occurs over most of the shocks, $F_{\text {tot }} \approx 1.0$, and $F_{\mathrm{esc}}>F_{\mathrm{adv}}$. Only once $\theta \gtrsim 90^{\circ}$ does the efficiency drop. At $\theta=0^{\circ}, F_{\text {tot }}=0.992$, while $F_{\text {tot }}=0.9$ and 0.5 at $\theta=116^{\circ}$ and $125^{\circ}$, respectively. Fig. 3e) shows the same quantities for the $\mathrm{O}$ shock. The same general behaviour is seen, though the shock stays efficient out to slightly higher values of $\theta$ (in this case $F_{\text {tot }}=0.9$ and 0.5 at $\theta=120^{\circ}$ and $126^{\circ}$ ). Our results can be compared against Fig. 10 in Blasi et al. (2005) where these quantities are shown as a function of the shock Mach number.

The maximum electron Lorentz factor is shown in Fig. 3f) for the two shocks. Both shocks have values of $\gamma_{\text {max, }} \approx 5 \times 10^{6}$ on the line-of-centres, and this value drops only slightly as $\theta$ increases. Only once past $\theta \approx 100^{\circ}$ does it begin to drop more rapidly. Thus the assumption of a constant value of $\gamma_{\text {max,e }}$ in our previous work (Dougherty et al. 2003; Pittard et al. 2006; Pittard \& Dougherty 2006) was a reasonably good one. The value of $\gamma_{\max , \mathrm{e}}$ in our models is strongly dependent on $D$ and $B_{0}$.

\subsubsection{The particle distributions}

Figs. 4 and 5 show the distributions of the thermal and nonthermal particles immediately downstream of the shock. In each figure the top two lines are the proton distributions, while the bottom two are the electron distributions. The particle distributions are shown for the WR shock (solid line) and the $\mathrm{O}$ shock (dashed line). Fig. 4 shows the distributions for $\theta=0^{\circ}$, while Fig. 5 shows them for $\theta=110^{\circ}$. In all cases the distributions clearly show strong shock modification, with most of the energy pushed towards the highest momenta. As previously noted, the maximum proton momentum is lower at the $\mathrm{O}$ shock than at the WR shock. The positions of the thermal peak also indicates the effect of cooler downstream thermal particles for modified shocks.

The cooling timescale for electrons $\left(t_{\text {cool }}=E /|\dot{E}|\right)$ for the various energy-loss mechanisms is shown in Fig. 6 . The electrons are assumed to be located near the apex of the WCR (specifically, they are immediately behind the WR shock at $\left.\theta=0.5^{\circ}\right)$. The cooling time is dominated by different mechanisms in different energy ranges. At the lowest energies, losses due to Coulomb scattering dominate, whereas inverse Compton cooling takes over for $E \gtrsim 10 \mathrm{MeV}$. At the highest energies shown inverse Compton cooling loses its dominance as its cross section reduces and synchrotron cooling becomes dominant at energies above $\sim 1 \mathrm{TeV}$. The relative strength of the inverse Compton and synchrotron cooling depends on a number of the model parameters, including $L_{\mathrm{WR}}, L_{\mathrm{O}}, D$, the pre-shock magnetic field, $B_{0}$, and the amount of compression/amplification of the magnetic field.

The downstream cooling of the non-thermal electron distribution from the WR shock at $\theta=0.5^{\circ}$ is shown in Fig. 7. Inverse Compton cooling dominates the cooling of the high energy electrons, while coulombic cooling dominates at low energies. The properties of the distributions are noted in Table 2. Because the post-shock tangential velocity is low (the wind collides almost normal to the shock) it takes a long time for the streamline to increase its value of $\theta$ (which it can do only in $1^{\circ}$ steps). By the time of the final distribution shown the particles have flowed downstream a total distance 

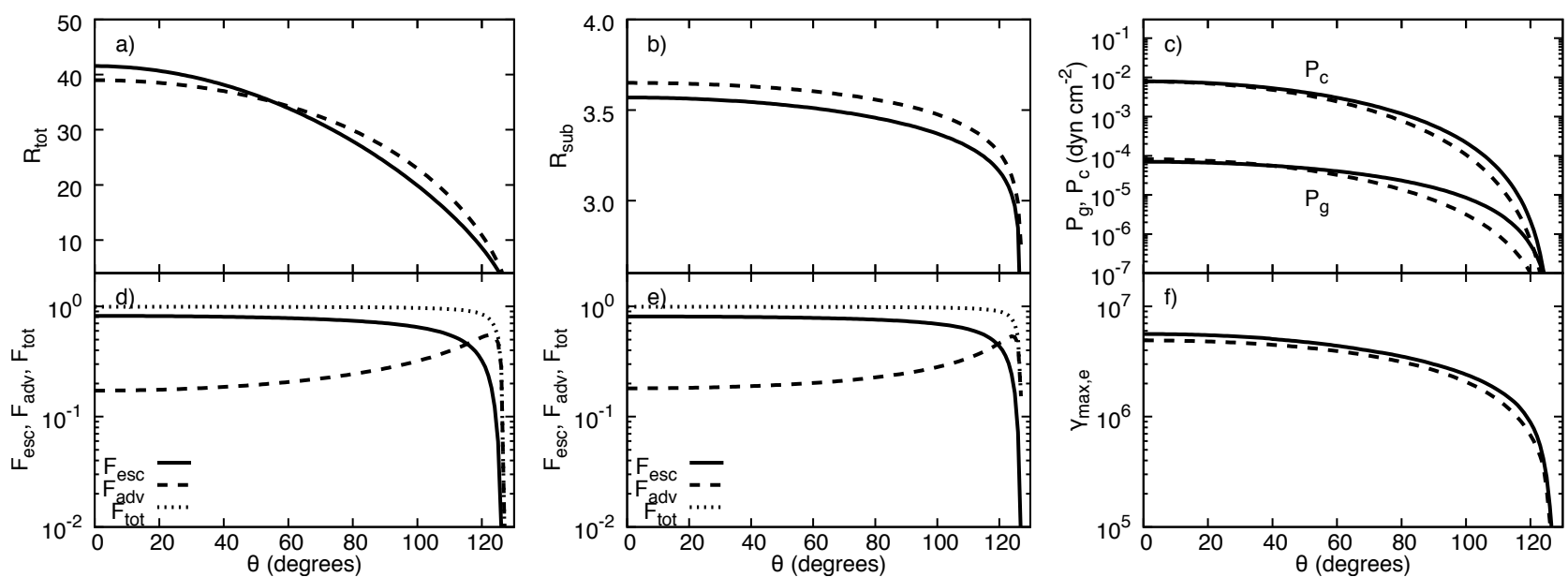

Figure 3. Selected quantities along the $\mathrm{CD}$ as a function of the angle $\theta$ from the secondary star. Panels a-c) show the total compression ratio of the shock, the compression ratio of the sub-shock, and the post-shock pressure from non-thermal $\left(P_{\mathrm{c}}\right)$ and thermal $\left(P_{\mathrm{g}}\right)$ particles, respectively. Panel d) shows the advected $\left(F_{\text {adv }}\right)$, escaping $\left(F_{\text {esc }}\right)$, and total non-thermal particle flux, normalised to the incoming kinetic energy flux, for the WR-star shock. Panel e) shows the equivalent for the O-star shock. Panel f) shows the maximum Lorentz factor of the non-thermal electrons from each shock. In panels a-c) and f) the solid line indicates the properties for the WR-star shock, while the dashed line indicates the properties for the O-star shock.

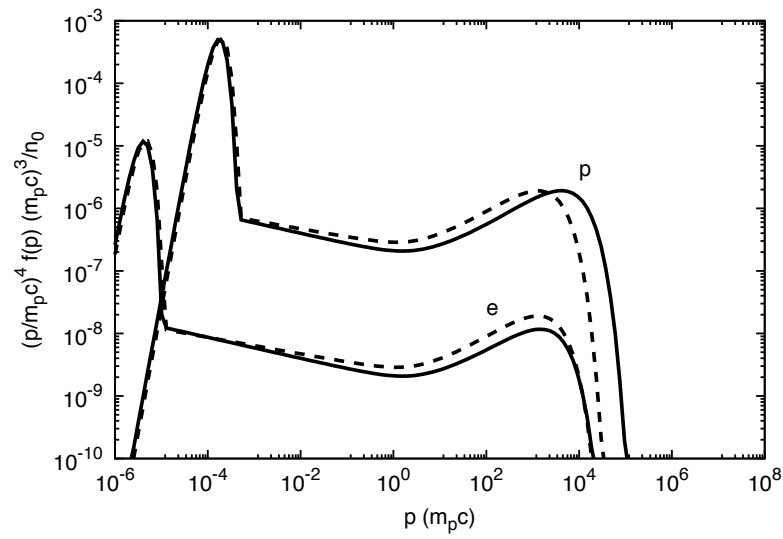

Figure 4. The proton and electron distributions for the WR shock (solid line) and O shock (dashed line) for $\theta=0^{\circ}$. For both shocks $n_{0}=1.3 \times 10^{5} \mathrm{~cm}^{-3}$. The thermal peaks are visible at low momenta.

of $0.015 \mathrm{D}$, taking $1.3 \times 10^{7} \mathrm{~s}$ to do so. In the code, the final distribution shown is actually the $158^{\text {th }}$ distribution stored along this streamline (i.e., the cooling is resolved very well), and a total of 172 distributions are calculated and stored along this streamline.

\subsubsection{The non-thermal emission}

Fig. 8 shows the non-thermal emission from our standard model. The inverse Compton emission is dominant for $E \lesssim 1 \mathrm{GeV}$, while $\pi^{0}$-decay emission becomes comparable in strength at higher energies. The emission from relativistic bremsstrahlung is always at least an order of magnitude fainter than the inverse Compton emission. Both shocks contribute roughly equally to the inverse Compton emission, though the relativistic bremsstrahlung and $\pi^{0}$-decay emission from the WR-star shock is about twice as bright as

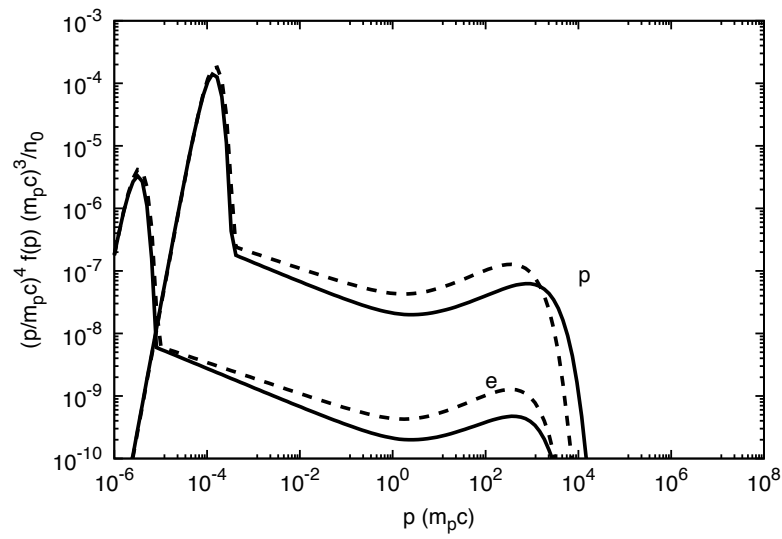

Figure 5. The proton and electron distributions for the WR shock (solid line) and $\mathrm{O}$ shock (dashed line) for $\theta=110^{\circ}$. For the WR shock $n_{0}=2.0 \times 10^{4} \mathrm{~cm}^{-3}$, while for the $\mathrm{O}$ shock $n_{0}=$ $4.2 \times 10^{3} \mathrm{~cm}^{-3}$

Table 2. The properties of the distributions shown in Fig. 7. The distributions are numbered from 1 to 8 , with the amount of cooling increasing with the distribution index. The value of $\theta$, the arc-length along the $\mathrm{CD}$ from the stagnation point, and the elapsed time since the shock are noted.

\begin{tabular}{llll}
\hline Index & $\theta\left(^{\circ}\right)$ & $l / D$ & $t(\mathrm{~s})$ \\
\hline 1 & 0.5 & $1.0 \times 10^{-6}$ & 2670 \\
2 & 0.5 & $6.5 \times 10^{-6}$ & $1.7 \times 10^{4}$ \\
3 & 0.5 & $3.6 \times 10^{-5}$ & $9.4 \times 10^{4}$ \\
4 & 0.5 & $1.5 \times 10^{-4}$ & $4.0 \times 10^{5}$ \\
5 & 0.5 & $6.1 \times 10^{-4}$ & $1.6 \times 10^{6}$ \\
6 & 0.5 & $9.6 \times 10^{-4}$ & $2.5 \times 10^{6}$ \\
7 & 1.5 & $4.3 \times 10^{-3}$ & $7.4 \times 10^{6}$ \\
8 & 3.5 & 0.015 & $1.3 \times 10^{7}$ \\
\hline
\end{tabular}




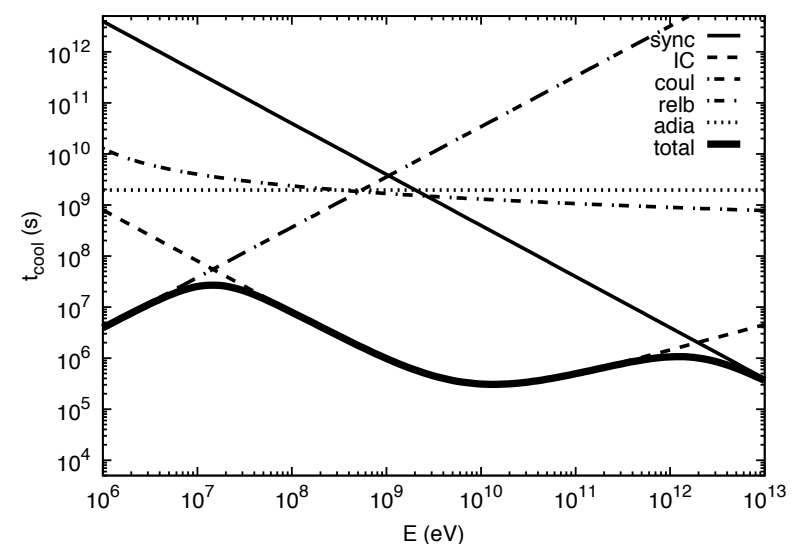

Figure 6. The cooling time $t_{\text {cool }}=E /|\dot{E}|$ as a function of electron energy for electrons located immediately post-WR-shock for $\theta=0.5^{\circ}$ in the "standard" model with $D=2 \times 10^{15} \mathrm{~cm}$. The parameters are $n_{\mathrm{e}}=5 \times 10^{5} \mathrm{~cm}^{-3}, T=4 \times 10^{4} \mathrm{~K}, B=0.01 \mathrm{G}, R=1.5 \times 10^{15} \mathrm{~cm}$, $v=v_{\mathrm{CD}}=0.8 \mathrm{~km} \mathrm{~s}^{-1}$. Coulomb cooling dominates at low energies, synchrotron cooling dominates at high energies, and inverse Compton cooling dominates at intermediate $(10 \mathrm{MeV}-2 \mathrm{TeV})$ energies.

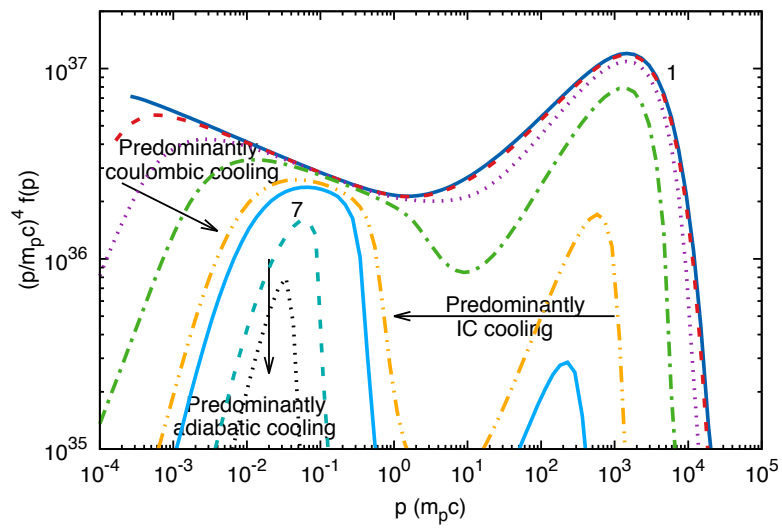

Figure 7. The downstream cooling of the electron distribution of the WR shock for $\theta=0.5^{\circ}$. The immediate post-shock distribution is the top-most line (labelled " 1 "), and the distribution shifts downwards and inwards with increasing cooling. Some properties of each distributions are noted in Table $2 . D=2 \times 10^{15} \mathrm{~cm}$.

that from the O-star shock. The O-star shock has brighter inverse Compton emission at energies above $10 \mathrm{MeV}$ than the WR-star shock. The signature of strong shock modification is visible as the curvature in the inverse Compton and relativistic bremsstrahlung emission which leads to increased flux at higher energies. Detection of such curvature from actual systems would indicate strong shock modification.

\subsection{Effect of binary separation and downstream cooling}

We now examine how the non-thermal particle distributions and the resulting emission changes when the stellar separation, $D$, is altered.

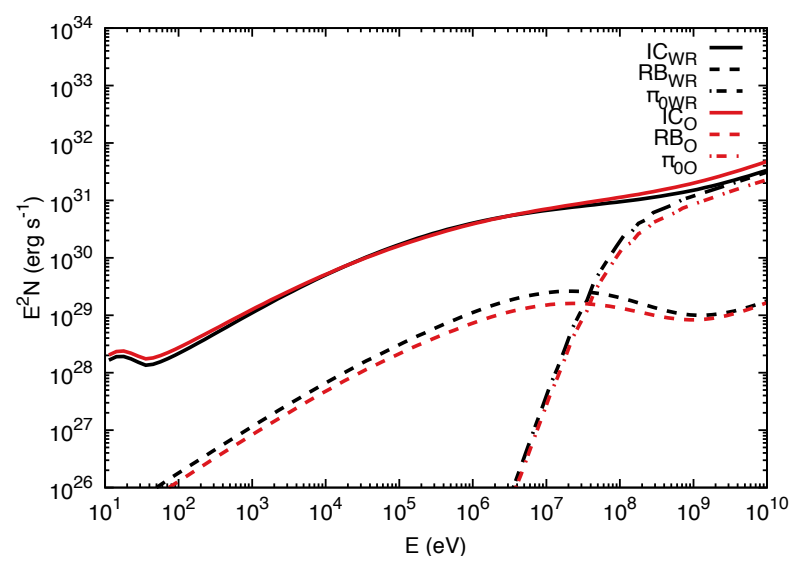

Figure 8. The non-thermal emission from each shock from our standard model. $D=2 \times 10^{15} \mathrm{~cm}$ and $\phi=90^{\circ}$. The black lines show the emission from the WR-star shock, while the red lines show it from the O-star shock.

\subsubsection{Expected scaling}

If the cooling timescale of the post-shock thermal particles $\left(t_{\text {cool,th }}\right)$ is long compared to their dynamical timescale to flow out of the system $\left(t_{\text {dyn }}\right)$, then the WCR behaves selfsimilarly, and its volume $V$ scales as $D^{3}$. In such circumstances the total emission from thermal particles, with number density $n_{\mathrm{th}}$, scales as $L_{\mathrm{th}} \propto n_{\mathrm{th}}^{2} V \propto D^{-1}$ (Stevens et al. 1992).

We now consider how the non-thermal emission should scale. The non-thermal particle density, $n_{\mathrm{NT}}$, scales as $D^{-2}$. If the non-thermal particles also do not strongly cool (i.e. their cooling timescale $t_{\text {cool, NT }} \lesssim t_{\text {dyn }}$ ), then they fill the WCR, and so the volume that they occupy scales as $D^{3}$. For the inverse Compton emission, the number density of stellar photons, $n_{\mathrm{ph}}$, also scales as $D^{-2}$, so we expect $L_{\mathrm{IC}} \propto n_{\mathrm{NT}} n_{\mathrm{ph}} V \propto D^{-1}$. We also expect the relativistic bremsstrahlung and the $\pi^{0}$ decay emission to both scale as $n_{\mathrm{th}} n_{\mathrm{NT}} V \propto D^{-1}$.

Now consider the situation where the thermal gas in the WCR is largely adiabatic but where there is very rapid cooling of the non-thermal particles. As noted by Hamaguchi et al. (2018), the cooling length $l_{\text {cool,NT }} \propto D^{2}$, so the "volume" that the non-thermal electrons occupy prior to being cooled below some energy limit $\left(V_{\mathrm{NT}}<V\right)$ is given by the area of the shocks $\left(A \propto D^{2}\right)$ multiplied by the cooling length: i.e. $V_{\mathrm{NT}}=A l_{\text {cool,NT }} \propto D^{4}$. In such cases we expect the nonthermal emission to scale as $D^{0}$.

We expect $p_{\max }$ to be independent of $D$, since $p_{\max } \propto$ $r_{\text {shk }} B_{0}$, with $r_{\text {shk }} \propto D$ and $B_{0} \propto U_{\mathrm{B}}^{1 / 2} \propto U_{\mathrm{KE}}^{1 / 2} \propto \rho_{0}^{1 / 2} \propto D^{-1}$. On the other hand, $p_{\text {max,e }}$, may depend on the strength of the inverse Compton cooling. By balancing the rate of energy gain through DSA with the rate of energy loss through inverse Compton cooling, we find that in such cases $p_{\text {max,e }} \propto$ $r_{\text {shk }} B_{0}^{1 / 2} \propto D^{1 / 2}$ (see, e.g., Pittard et al. 2006). We find the same scaling of $p_{\text {max,e }}$ with $D$ if instead $p_{\text {max,e }}$ depends on synchrotron cooling.

\subsubsection{The particle distributions}

Fig. 9 compares the on-axis $\left(\theta=0^{\circ}\right)$ post-shock particle distributions for the WR shock for $D=2 \times 10^{14} \mathrm{~cm}$ and 


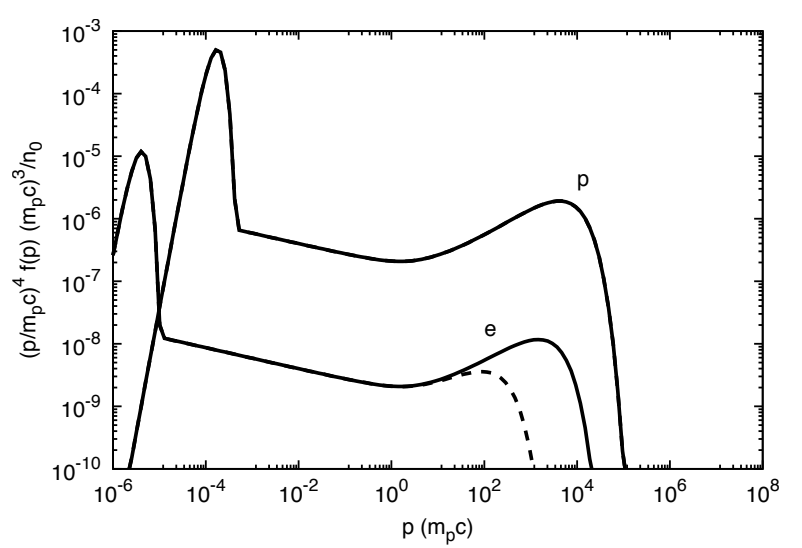

Figure 9. The proton and electron distributions for the on-axis WR shock as a function of $D$. The solid lines have $D=2 \times 10^{15} \mathrm{~cm}$, while the dashed lines have $D=2 \times 10^{14} \mathrm{~cm}$. The solid and dashed lines are coincident for the proton distributions when normalised by the pre-shock number density.

$D=2 \times 10^{15} \mathrm{~cm}$, normalized to the pre-shock number density. Because $M_{0 \perp}, u_{0 \perp}$ and $p_{\max }$ are all independent of $D$, the (normalized) proton distributions are identical for the two distances. However, the electron distribution is cut off at a lower maximum momentum when $D=2 \times 10^{14} \mathrm{~cm}$ due to the enhanced inverse Compton cooling. We find that $p_{\max } \propto D^{0}$ and $p_{\text {max,e }} \propto D^{1 / 2}$ as expected.

\subsubsection{The non-thermal emission}

Before we examine the effect on the non-thermal emission of varying $D$, it is helpful to examine the effect of downstream cooling on the non-thermal emission for our standard model $\left(D=2 \times 10^{15} \mathrm{~cm}\right)$. This is indicated in Fig. 10, where the difference between the red and black lines shows the effect of including all the cooling processes noted in Sec. 2.4 versus considering only adiabatic cooling). The high energy electrons cool strongly due to IC emission while the lower energy electrons cool through coulombic collisions. Cooling of the non-thermal electrons reduces the inverse Compton and relativistic bremsstrahlung emission at $\mathrm{GeV}$ energies by $\sim 1$ dex. In contrast, there is little cooling of the non-thermal protons, as evidenced by the almost unchanged $\pi^{0}$-decay emission.

The inverse Compton emission from particles along the single $\theta=0.5^{\circ}$ streamline from the WR-shock is approximately flat in the $E^{2} N$ spectrum at $10 \mathrm{MeV}$, with an upward curvature with increasing energy due to the strongly modified nature of the shock. In contrast, Fig. 10 shows that there is a slight rise in the inverse Compton emission at $10 \mathrm{MeV}$ (see the solid black line). This rise is caused by higher energy breaks in the electron distributions along the other streamlines.

The effect of downstream cooling on the non-thermal emission for a model with reduced binary separation $(D=$ $\left.2 \times 10^{14} \mathrm{~cm}\right)$ is shown in Fig. 11. Compared to Fig. 10 we see that the effect of cooling has strengthened, as expected given the reduced separation.

Fig. 12 shows the effect of binary separation on the nonthermal emission if only adiabatic cooling is applied to the

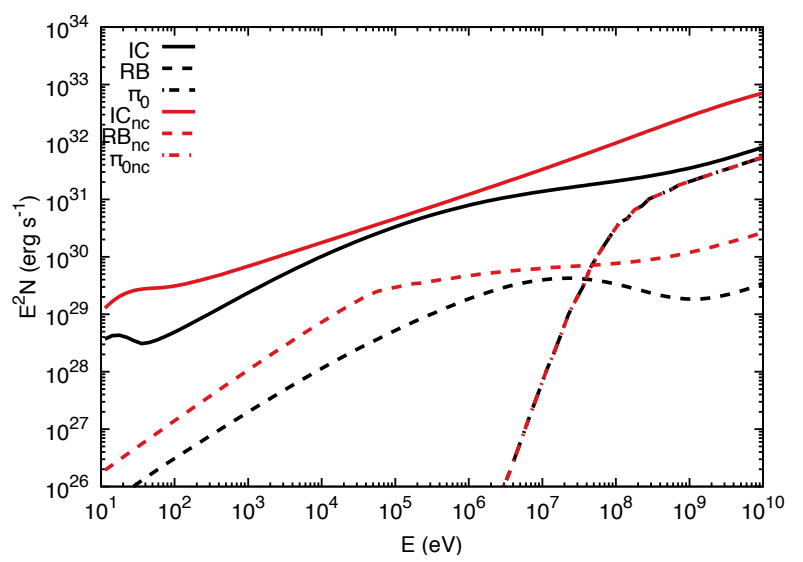

Figure 10. The effect of cooling on the downstream non-thermal particles and their subsequent emission. The red lines include only adiabatic cooling, while the black lines include all cooling processes considered in this work (adiabatic, coulombic, inverse Compton, synchrotron and relativistic bremsstrahlung cooling for the electrons; adiabatic and proton-proton cooling for the protons). $D=2 \times 10^{15} \mathrm{~cm}$ and $\phi=90^{\circ}$.

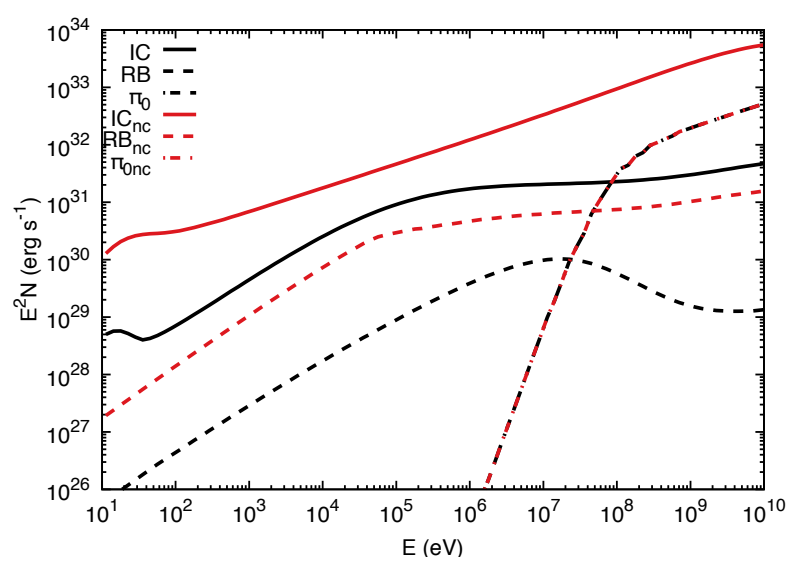

Figure 11. As Fig. 10 but for $D=2 \times 10^{14} \mathrm{~cm} . \phi=90^{\circ}$.

downsteam non-thermal particles. We see that all emission processes scale as $D^{-1}$, as expected.

Finally, Fig. 13 shows the effect of binary separation on the non-thermal emission if cooling is fully applied to the downsteam non-thermal particles. We now find that the previous $D^{-1}$ scaling of the inverse Compton and relativistic bremsstrahlung emission disappears and the change with $D$ becomes much reduced. However, the emission from $\pi^{0}$ decay still varies strongly (and almost as $D^{-1}$ ), again illustrating that the non-thermal protons do not undergo strong downstream cooling.

This behaviour contrasts with some other modelling work in the literature. For instance, Figs. 12 and 13 in Reimer et al. (2006) show the relativistic bremsstrahlung and $\pi^{0}$-decay $\gamma$-ray spectra from the acceleration region in their model scaling roughly as $D^{-4}$. Unfortunately the scaling behaviour of the total emission (from the acceleration and convection region combined) is not shown. Likewise, the flux variations in Models A-C in Fig. 10 of Reitberger et al. (2014b) show no sign of becoming independent of $D$, despite the close-ish separations. We note that Reitberger et al. use 


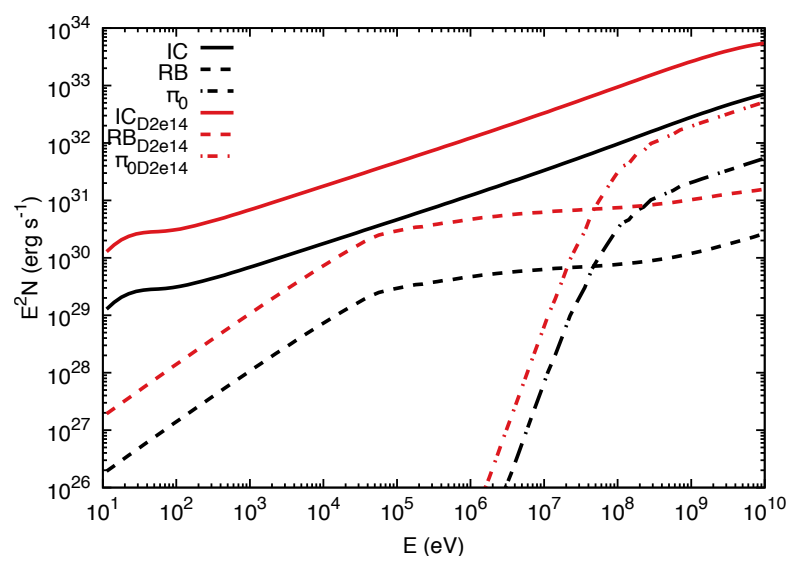

Figure 12. The effect of binary separation on the non-thermal emission. Only adiabatic cooling of the downstream non-thermal particles has been applied. The black lines are for $D=2 \times 10^{15} \mathrm{~cm}$ and the red lines are for $D=2 \times 10^{14} \mathrm{~cm} . \phi=90^{\circ}$.

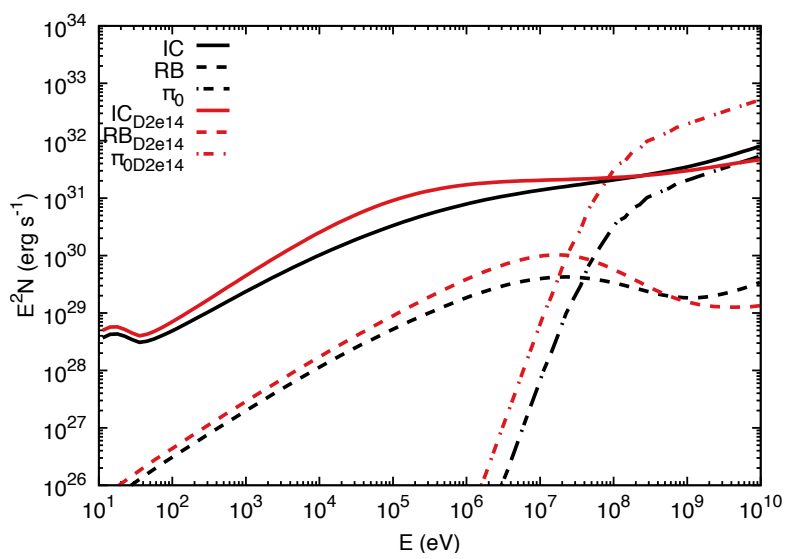

Figure 13. The effect of binary separation on the non-thermal emission. Cooling of the downstream non-thermal particles has been fully applied. The black lines are for $D=2 \times 10^{15} \mathrm{~cm}$ and the red lines are for $D=2 \times 10^{14} \mathrm{~cm} . \phi=90^{\circ}$.

a fixed size for their computational volume. A plausible explanation for their results is that a a greater fraction of the total emission was "missed" from the model with the wider stellar separation.

Fig. 14 shows how the inverse Compton emission changes with stellar separation. At low energies and large separations the slope of the lines is $\approx-1$, indicating that the responsible particles fill the WCR (i.e. they are not strongly cooling as they flow away from the shock). However, cooling becomes increasingly important as $D$ decreases. The emission at $10^{3}$ and $10^{5} \mathrm{eV}$ no longer scales as $D^{-1}$, but still scales to some inverse power. In contrast, at the higher energies we find that the flux reaches a maximum at an intermediate value of $D$, and then decreases as $D$ becomes still smaller. This is caused by $p_{\text {max,e }}$ decreasing with decreasing $D$, which has the knock-on effect that the fluxes begin to be affected by the exponential cut-off of the non-thermal electron particle distribution.

Fig. 15 shows the effect of changing the binary separation on the $\pi^{0}$-decay emission at $10^{9} \mathrm{eV}$. At large $D$ we again see that the flux scales as $D^{-1}$, but again witness a

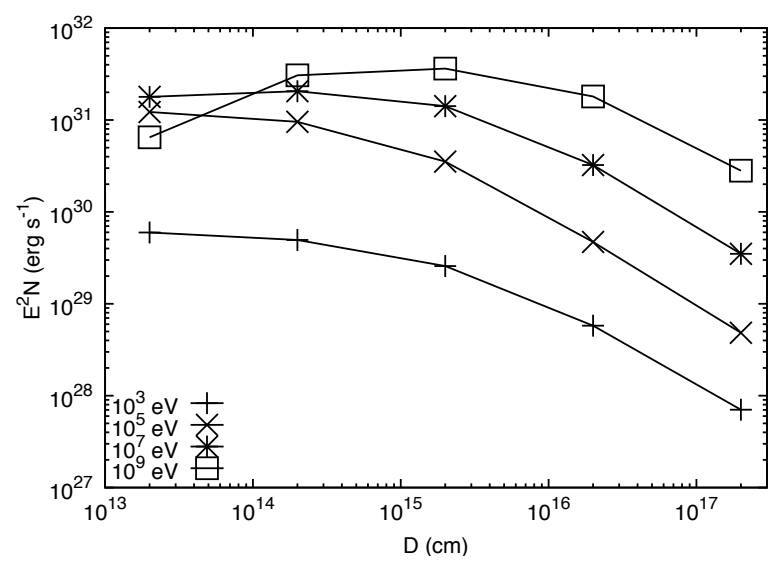

Figure 14. The effect of binary separation on the inverse Compton emission. Data points are shown at four energies: $10^{3}, 10^{5}$, $10^{7}$, and $10^{9} \mathrm{eV} . \phi=90^{\circ}$

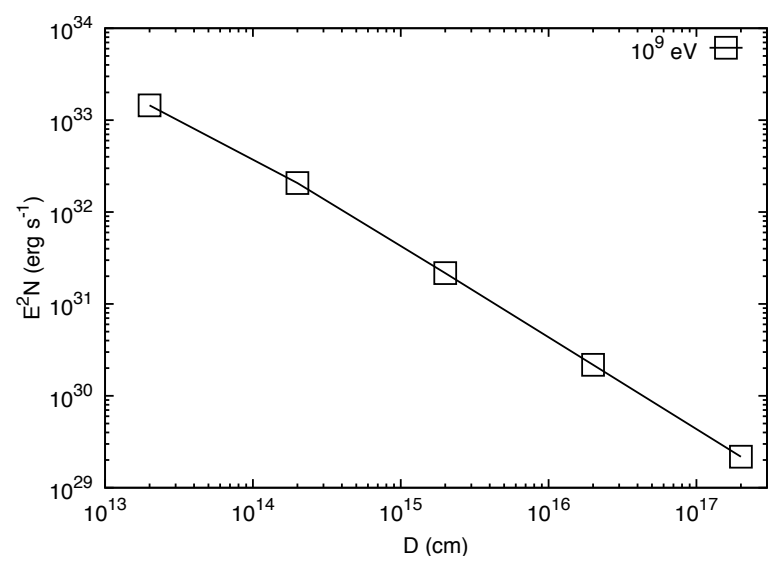

Figure 15. The effect of the binary separation on the $\pi^{0}$-decay emission at $10^{9} \mathrm{eV}$.

turndown in this slope as $D$ decreases. It is clear that cooling of the downstream non-thermal protons starts to become significant for $D \lesssim 10^{14} \mathrm{~cm}$.

\subsubsection{Comparison to observations}

At the time of writing the strongest evidence for orbital variability of non-thermal X-ray and $\gamma$-ray emission comes from Fermi observations of $\eta$ Carinae. Balbo \& Walter (2017) find that of the two emission components seen by Fermi, the flux of the low-energy $(0.3-10 \mathrm{GeV})$ component is modulated by the orbit, being stronger near periastron and weaker at apastron. Overall, it varies by less than a factor of 2 . This component is likely inverse Compton emission, and it is probably not significantly affected by photon-photon absorption. On the other hand, the high-energy $(10-300 \mathrm{GeV})$ component varies by a factor of $3-4$ and is different during the two periastrons that are observed (see their Fig. 5). This component is likely emission from $\pi^{0}$-decay and will be strongly affected by photon-photon absorption.

In contrast, Fig. 14 shows that at $E=10^{9} \mathrm{eV}$, the inverse Compton flux in our model increases by a factor of $\approx 5$ when $D$ increases from $2-20 \times 10^{13} \mathrm{~cm}$ (for $\eta$ Car, $D=2.3-44 \times$ $10^{13} \mathrm{~cm}$ between periastron and apastron). Thus the flux is 
our model behaves the opposite way to the observed emission from $\eta$ Car, which decreases with increasing $D$. It will be interesting to see if these differences can be reconciled with a dedicated application of our model to $\eta$ Car (the modelling in Balbo \& Walter (2017) is able to reproduce the variation, to first order).

\subsection{Effect of observing angle}

We now examine the effect on the non-thermal emission of changing the observing angle. Because no absorption processes are included in the current model only the anisotropic nature of the inverse Compton emission affects the observed non-thermal emission. This is shown in Fig. 16. Since our model is axisymmetric, changing only the observing angle covers any orientation of the system relative to the observer. At an observing angle $\phi=0^{\circ}$ the secondary star is in front, quadrature is at $\phi=90^{\circ}$, and the primary star is in front at $\phi=180^{\circ}$. The strongest emission occurs when the secondary star is in front, while the weakest emission occurs when the primary star is in front. This agrees with expectations since the secondary star is the major source of incident photons and is closest to the WCR.

There is not much change in the emission when one of the stars is within $\sim 30^{\circ}$ from being directly in front. This is likely due to the fact that the asymptotic half-opening angle of the WCR for $\eta=0.1$ is $\approx 50^{\circ}$ (Pittard \& Dawson 2018), so that the line of sight is still within the shock cone for this range of viewing angle. We find that it is only when the line of sight moves outside of the shock cone that the emission become more sensitive to changes in viewing angle.

The overall variation is about a factor of 3.5 (as measured at $E=1 \mathrm{MeV}$ ). This is much lower than in other work (e.g. Reimer et al. 2006; Dubus et al. 2008) which we attribute to the way that the WCR wraps around the secondary star in our model. Although the stars are almost point-like on this scale the spatial distribution of the nonthermal particles is anything but. The part of the wind-wind collision region that experiences a photon flux from the secondary star that is within a factor of 3 of the peak flux that occurs at the apex extends to $\theta \approx 73^{\circ}$. This region covers 36 per cent of the sky as seen from the O-star and has the effect of substantially reducing the change in IC emission with the viewing angle. The presence of the WR star also reduces the level of variability in our model.

\subsection{Effect of varying the magnetic field strength}

In the model we are free to set the strength of the pre-shock magnetic field. This is controlled through the value of $\zeta_{\mathrm{B}}$. Our standard model has $\zeta_{\mathrm{B}}=10^{-3}$. Fig. 17 shows the effect of changing $\zeta_{\mathrm{B}}$ within the range $10^{-4} \leq \zeta_{\mathrm{B}} \leq 10^{-2}$. Lower values of $\zeta_{\mathrm{B}}$ imply a lower pre-shock magnetic flux density, $B_{0}$, which in turn reduces the maximum momentum that the non-thermal protons reach $\left(p_{\max } \propto B_{0} \propto \zeta_{\mathrm{B}}^{1 / 2}\right)$. This can dramatically affect the efficiency of the DSA, and can significantly alter the shape of the non-thermal particle spectrum.

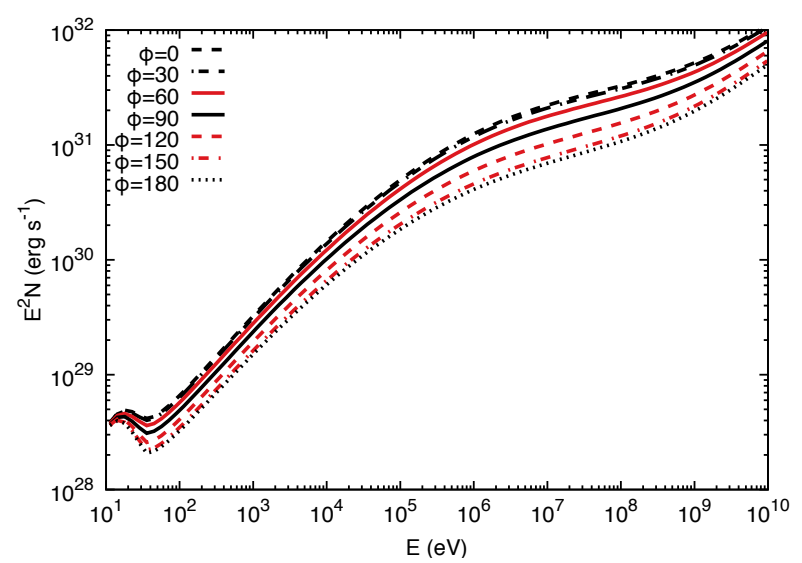

Figure 16. The effect of the observing angle on the inverse Compton emission. The standard model has $\phi=90^{\circ}$. At $\phi=0^{\circ}$ the secondary star is in front, while the primary star is in front when $\phi=180^{\circ} . D=2 \times 10^{15} \mathrm{~cm}$.

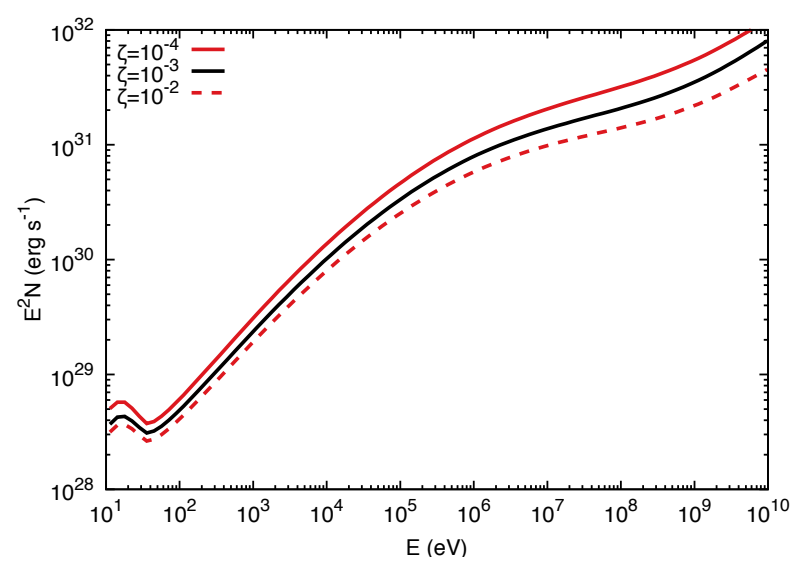

Figure 17. The effect of the pre-shock magnetic flux density on the inverse Compton emission. The standard model has $\zeta_{\mathrm{B}}=10^{-3}$. $D=2 \times 10^{15} \mathrm{~cm}$ and $\phi=90^{\circ}$.

\subsection{Effect of varying the injected particle momentum}

A second free parameter in the DSA model is $\chi_{\text {inj, }}$ which controls the momentum of the injected particles. The effect on the post-WR-shock non-thermal particle distributions of setting $\chi_{\text {inj }}=2.0$ is shown in Fig. 18. Both distributions see an increase in the number of non-thermal particles from the thermal peak up to momenta of order $m_{p} c$, but show little change above this. The variation in the proton distribution is comparable to the differences seen when different methods are used to calculate the DSA (see, e.g., Fig. 1 in Caprioli et al. 2010).

The effect on the non-thermal emission of varying $\chi_{\text {inj }}$ is shown in Fig. 19. We see that the inverse Compton emission becomes softer as $\chi_{\text {inj }}$ decreases. This is because more electrons with $p<\mathrm{m}_{\mathrm{p}} \mathrm{c}$ (i.e with $\gamma \lesssim 10^{3}$ ) take part in the DSA. Because the $\pi^{0}$-decay emission is produced by non-thermal protons that exceed the threshold energy of $1.22 \mathrm{GeV}$ (see App. B3), it is not sensitive to the changes in the nonthermal proton distribution that occur for $p<\mathrm{m}_{\mathrm{p}} \mathrm{c}$. 


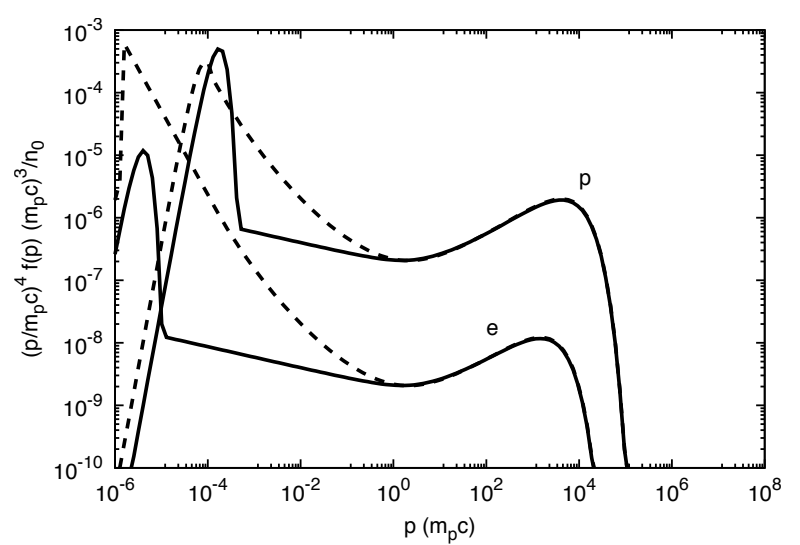

Figure 18. The effect of the injected particle momentum on the proton and electron distributions for the on-axis WR shock as a function of $\chi_{\mathrm{inj}}$. The solid lines have $\chi_{\mathrm{inj}}=3.5$ (the standard model), while the dashed lines have $\chi_{\text {inj }}=2.0 . D=2 \times 10^{15} \mathrm{~cm}$.

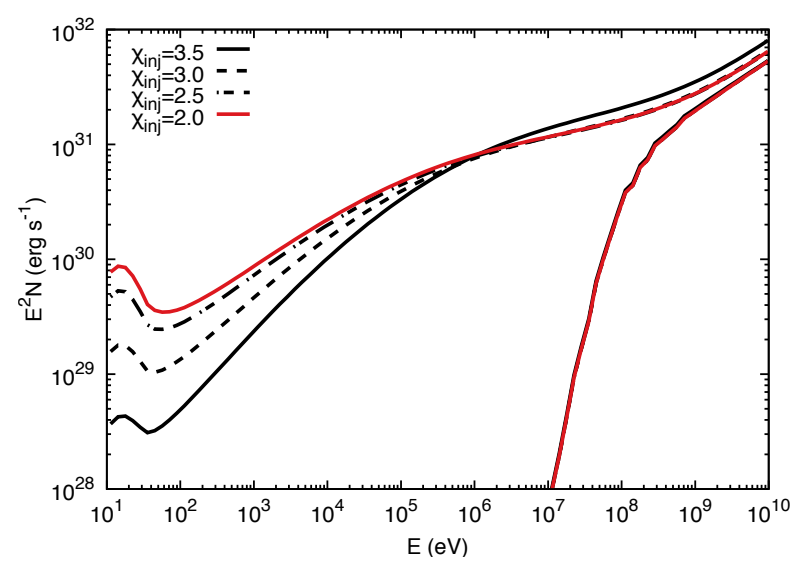

Figure 19. The effect of the injected particle momentum on the non-thermal inverse Compton and $\pi^{0}$-decay emission. The standard model has $\chi_{\mathrm{inj}}=3.5 . D=2 \times 10^{15} \mathrm{~cm}$ and $\phi=90^{\circ}$.

\subsection{Effect of varying the wind momentum ratio}

Our standard model has a wind momentum ratio $\eta=0.1$. To examine the resulting non-thermal emission when $\eta=0.01$ we reduce the mass-loss rate of the secondary star. This change means that there is less energy in the winds that can ultimately be turned into non-thermal emission. However, several effects act together. Firstly, while less of the primary wind is shocked, a greater fraction of the (weaker) secondary wind is shocked. Secondly, the WCR moves closer to the secondary star. Since the wind speeds have not changed this means that the on-axis pre- and post-shock density of the primary and secondary wind both decline, as does the pre-shock magnetic flux density. However, the photon flux from the secondary star at the apex of the WCR increases. The maximum non-thermal proton momentum at the on-axis point of the WR shock remains unchanged $\left(p_{\max }=8.5 \times 10^{3} \mathrm{~m}_{\mathrm{p}} \mathrm{c}\right)$, but reduces at the on-axis point of the $\mathrm{O}$ shock to $p_{\max }=8.5 \times 10^{2} \mathrm{~m}_{\mathrm{p}} \mathrm{c}$. The maximum nonthermal electron momentum at the apex of both shocks is $p_{\text {max }, \mathrm{e}}=340 \mathrm{~m}_{\mathrm{p}} \mathrm{c}$, corresponding to $\gamma_{\max , \mathrm{e}}=6.3 \times 10^{5}$ and $\mathrm{a}$ reduction of about a factor of 8 from the standard model.

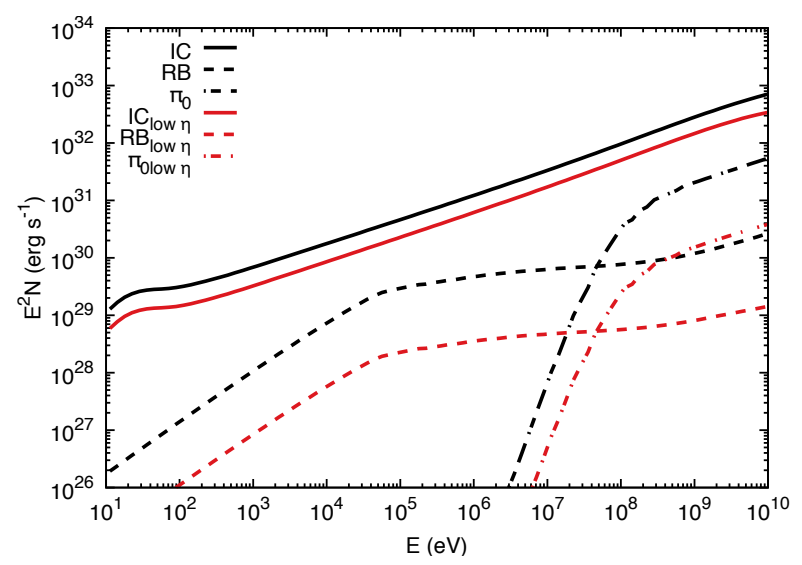

Figure 20. The effect of the wind momentum ratio, $\eta$, on the non-thermal emission, if only adiabatic cooling of the non-thermal particles takes place downstream of the shock. The black lines are for $\eta=0.1$, and the red lines are for $\eta=0.01$. $D=2 \times 10^{15} \mathrm{~cm}$ and $\phi=90^{\circ}$.

Finally, the WCR changes shape through a reduction in the asymptotic opening angle.

Fig. 20 shows the effect of reducing the wind momentum ratio, $\eta$, on the emission that would result if the nonthermal particles were allowed only to undergo adiabatic cooling downstream of the shocks. It shows that all three types of emission are reduced when $\dot{M}_{\mathrm{O}}$ is reduced. Thus the reduced strength of the combined winds dominates over other factors (e.g., the enhanced photon flux from the secondary star at the apex of the WCR). The fact that there is less of a reduction to the inverse Compton emission than to the relativistic bremsstrahlung and $\pi^{0}$-decay emission is consistent with the enhanced secondary star photon flux at the WCR somewhat offsetting the other factors noted above that act to reduce the flux. The reduction in $\gamma_{\text {max,e }}$ as $\eta$ is reduced also affects the position of the high-energy turnover of the inverse Compton emission (not clearly visible in Fig. 20).

Fig. 21 shows the effect on the non-thermal emission of reducing $\eta$, with the cooling of the non-thermal particles downstream of the shocks fully applied. The greater reduction in the inverse Compton emission with $\eta$ compared to the case where the downstream non-thermal particles undergo only adiabatic cooling (see Fig. 20) highlights the enhanced secondary star photon flux in this case.

\section{SUMMARY AND CONCLUSIONS}

We have created a new model for the non-thermal emission from colliding-wind binaries. Our model uses the Blasi et al. (2005) model to solve the diffusive shock acceleration of the particles at the global shocks. We confirm earlier work that DSA is very efficient with our chosen parameters and assumptions, leading to significantly modified shocks. This is the first CWB model that self-consistently includes shock modification.

We find a complicated dependence for the scaling of the non-thermal flux with the binary separation, $D$. If the nonthermal particles suffer little cooling when flowing downstream from the shocks the inverse Compton, relativistic bremsstrahlung and $\pi^{0}$-decay emission all scale as $D^{-1}$. This 


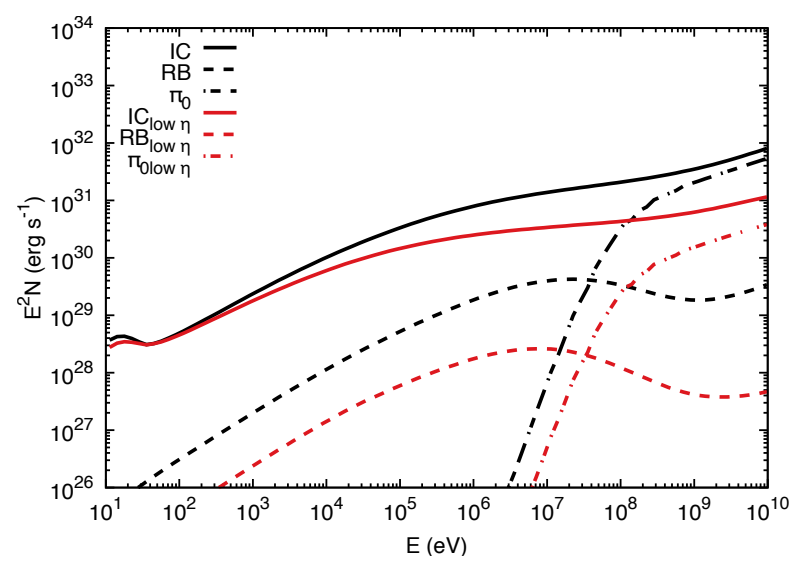

Figure 21. The effect of the wind momentum ratio, $\eta$, on the non-thermal emission. The black lines are for $\eta=0.1$, and the red lines are for $\eta=0.01 . D=2 \times 10^{15} \mathrm{~cm}$ and $\phi=90^{\circ}$.

occurs most readily at wide separations and/or from leptonic emission from lower energy particles. However, when $D$ decreases, the cooling of the non-thermal particles increases, and simple arguments indicate that the emission should plateau at a maximum value, becoming independent of $D$. The $\pi^{0}$-decay emission and the lower-energy inverse Compton emission behaves this way, but we observe more complicated behaviour for higher-energy inverse Compton emission where the emission actually peaks at an intermediate value of $D$ and thereafter declines as $D$ decreases further. This behaviour is caused by $p_{\text {max,e }}$ also decreasing with $D$. In real systems we may expect additional effects caused by variations in the pre-shock wind velocities with $D$.

We also find that the anisotropic inverse Compton emission shows only a moderate variation with viewing angle due to the spatial extent of the wind-wind collision. For a system with a wind momentum ratio of 0.1 we find that the variation with viewing angle is limited to a factor of $\approx 3.5$.

Reducing the wind momentum ratio from $\eta=0.1$ to $\eta=0.01$ (by reducing the value of $\dot{M}_{2}$ ) leads to a reduction in the non-thermal emission due to the weaker windwind collision, though the inverse Compton emission does not decline as much as the relativistic bremsstrahlung and $\pi^{0}$-decay emission because in our model the stellar photon flux at the apex of the WCR increases (however, in real systems the luminosity of the secondary star may reduce too).

The first application of our new model is presented in Mossoux et al. (2020), where it is compared against NuSTAR data on Cyg OB2 No.8A, a O6 I + O5.5 III system with a $21.9 \mathrm{~d}$ period and a slightly eccentric orbit $(e \sim 0.2)$. In future we will apply our model to other particle-accelerating CWB systems, such as $\eta$ Car, $\gamma^{2}$ Vel, and those in the catalogue of De Becker \& Raucq (2013).

This is an exciting time for research into the nonthermal X-ray and $\gamma$-ray emission from CWBs, with detections at $\mathrm{TeV}$ energies expected by the future Cherenkov Telescope Array (CTA; see Chernyakova et al. 2019). Future improvements to our model will include calculations of the thermal free-free and synchrotron emission, the creation of and emission from secondary particles, and the addition of free-free and photon-photon absorption. Radiative inhibition (Stevens \& Pollock 1994) and braking (Gayley, Owocki
\& Cranmer 1997), and orbital effects (Pittard 2009), will also be examined in future work.

\section{ACKNOWLEDGEMENTS}

We thank the referee for some useful suggestions which improved the quality of the paper. The calculations herein were performed on the DiRAC 1 Facility at Leeds jointly funded by STFC, the Large Facilities Capital Fund of BIS and the University of Leeds and on other facilities at the University of Leeds. Data for the figures in this paper are available from https://doi.org/XXX. GER was supported by grants AYA2016-76012-C3-1-P (Ministerio de Educación, Cultura y Deporte, España) and PIP 0338 (CONICET, Argentina).

\section{REFERENCES}

Abdo A.A., et al., 2010, ApJ, 723, 649

Aharonian F.A., Atoyan A.M., 2000, A\&A, 362, 937

Balbo M., Walter R., 2017, A\&A, 603, A111

Bednarek W., Pabich J., 2011, A\&A, 530, A49

Benaglia P., 2016, PASA, 33, 17

Benaglia P., Romero G.E., 2003, A\&A, 399, 1121

Benaglia P., Marcote B., Moldón J., Nelan E., De Becker M., Dougherty S.M., Koribalski B.S., 2015, A\&A, 579, A99

Berezinskii V.S., Bulanov S.V., Dogiel V.A., Ptuskin V.S., 1990, "Astrophysics of Cosmic Rays" (North-Holland, Amsterdam)

Blasi P., Gabici S., Vannoni G., 2005, MNRAS, 361, 907

Blomme R., Fenech D.M., Prinja R.K., Pittard J.M., Morford J.C., 2017, A\&A, 608, A69

Blomme R., et al., 2013, A\&A, 550, A90

Blumenthal G.R., Gould R.J., 1970, Rev. Mod. Phys., 42, 237

Brookes D.P., 2016, PhD thesis, The University of Birmingham

Cantó J., Raga A.C., Wilkin F.P., 1996, ApJ, 469, 729

Caprioli D., Blasi P., Amato E., Vietri M., 2009, MNRAS, 395, 895

Caprioli D., Kang H., Vladimirov A.E., Jones T.W., 2010, MNRAS, 407, 1773

Cerutti B., 2007, Master thesis, Ecole Nationale Supérieure de Physique de Grenoble, INPG

Cerutti B., 2010, PhD thesis "High-energy gamma-ray emission in compact binaries", Astrophysics, Université de Grenoble

Chernyakova M., et al., 2019, A\&A, 631, A177

Corcoran M.F., 2005, AJ, 129, 2018

Corcoran M.F., Hamaguchi K., Pittard J.M., Russell C.M.P., Owocki S.P., Parkin E.R., Okazaki A., 2010, ApJ, 725, 1528

Damineli A., et al., 2008, MNRAS, 384, 1649

De Becker M., Raucq F., 2013, A\&A, 558, A28

del Palacio S., Bosch-Ramon V., Romero G.E., Benaglia P., 2016, A\&A, 591, 139

Dougherty S.M., Beasley A.J., Claussen M.J., Zauderer B.A., Bolingbroke N.J., 2005, ApJ, 623, 447

Dougherty S.M., Pittard J.M., Kasian L., Coker R.F., Williams P.M., Lloyd H.M., 2003, A\&A, 409, 217

Dougherty S.M., Pittard J.M., 2006, in Proc. of the 8th European VLBI Network Symp., 49

Dougherty S.M., Williams P.M., Pollacco D.L., 2000, MNRAS, 316,143

Dubus G., Cerutti B., Henri G., 2008, A\&A, 477, 691

Dzib S.A., Rodríguez L.F., Loinard L., Mioduszewski A.J., OrtizLeón G.N., Araudo A.T., 2013, ApJ, 763, 139

Eichler D., Usov V., 1993, ApJ, 402, 271

Farnier C., Walter R., Leyder J.-C., 2011, A\&A, 526, A57

Gayley K.G., Owocki S.P., Cranmer S.R., 1997, ApJ, 475, 786 
Ginzburg V., Syrovatskii S., 1964, "The Origin of Cosmic Rays" (New York: Macmillan)

Grimaldo E., Reimer A., Kissmann R., Niederwanger F., Reitberger K., 2019, ApJ, 871, 55

Hamaguchi K., et al., 2007, ApJ, 663, 522

Hamaguchi K., et al., 2014, ApJ, 795, 119

Hamaguchi K., et al., 2018, Nature Astronomy, 2, 731

Heitler W., 1954, "Quantum Theory of Radiation"

H.E.S.S. Collaboration, Abdalla et al., 2020, arXiv:2002.02336

Kelner S.R., Aharonian F.A., Bugayov V.V., 2006, Phys. Rev. D, 74,034018

Khangulyan D., Aharonian F.A., 2005, in American Institute of Physics Conference Series, 745, High Energy Gamma-Ray Astronomy, ed. F.A. Aharonian, H.J. Völk, \& D. Horns, 359

Leyder J.-C., Walter R., Rauw G., 2008, A\&A, 477, L29

Leyder J.-C., Walter R., Rauw G., 2010, A\&A, 524, A59

Madura T.I., et al., 2013, MNRAS, 436, 3820

Manolakou K., Horns D., Kirk J.G., 2007, A\&A, 474, 689

Mehner A., Davidson K., Ferland G.J., Humphreys R.M., 2010, ApJ, 710, 729

Mossoux E., Pittard J.M., Rauw G., Nazé Y., 2020, A\&A, accepted (arXiv:2003.10262)

O'Connor E.P., Dougherty S.M., Pittard J.M., Williams P.M., 2005, in Massive Stars and High-Energy Emission in OB Associations, eds. G. Rauw, Y. Nazé, R. Blomme, \& E. Gosset, 81

Ohm S., Zabalza V., Hinton J.A., Parkin E.R., 2015, MNRAS, 449, L132

Okazaki A.T., Owocki S.P., Russell C.M.P., Corcoran M.F., 2008, MNRAS, 388, L39

Ortiz-León G., Loinard L., Rodríguez L.F., Mioduszewski A.J., Dzib S.A., 2011, ApJ, 737, 30

Parkin E.R., Pittard J.M., 2008, MNRAS, 388, 1047

Parkin E.R., Pittard J.M., Corcoran M.F., Hamaguchi K., Stevens I.R., 2009, MNRAS, 394, 1758

Parkin E.R., Pittard J.M., Corcoran M.F., Hamaguchi K., 2011, ApJ, 726, 105

Pittard J.M., 2009, MNRAS, 396, 1743

Pittard J.M., Corcoran M.F., 2002, A\&A, 383, 636

Pittard J.M., Dawson B., 2018, MNRAS, 477, 5640

Pittard J.M., Dougherty S.M., 2006, MNRAS, 372, 801

Pittard J.M., Dougherty S.M., Coker R.F., O'Connor E., Bolingbroke N.J., 2006, A\&A, 446, 1001

Pshirkov M.S., 2016, MNRAS, 457, L99

Reimer A., Pohl M., Reimer O., 2006, ApJ, 644, 1118

Reitberger K., Kissmann R., Reimer A., Reimer O., Dubus G., 2014a, ApJ, 782, 96

Reitberger K., Kissmann R., Reimer A., Reimer O., 2014b, ApJ, 789,87

Reitberger K., Reimer A., Reimer O., Takahashi H., 2015, A\&A, 577, A100

Reitberger K., Kissmann R., Reimer A., Reimer O., 2017, ApJ, 847,40

Reitberger K., Reimer O., Reimer A., Werner M., Egberts K., Takahashi H., 2012, A\&A, 544, A98

Rybicki G.B., Lightman A.P., 1979, "Radiative Processes in Astrophysics"

Sekiguchi A., Tsujimoto M., Kitamoto S., Ishida M., Hamaguchi K., Mori H., Tsuboi Y., 2009, PASJ, 61, 629

Stecker F.W., 1971, Cosmic Gamma Rays, Baltimore: Mono Book corp.

Stevens I.R., Blondin J.M., Pollock A.M.T., 1992, ApJ, 386, 265

Stevens I.R., Pollock A.M.T., 1994, MNRAS, 269, 226

Tavani M., et al., 2009, ApJL, 698, L142

Vila G.S., 2012, PhD thesis "Radiative models for jets in X-ray binaries", Universidad de Buenos Aires

Williams P.M., Dougherty S.M., Davis R.J., van der Hucht K.A., Bode M.F., Setia Gunawan D.Y.A., 1997, MNRAS, 289, 10
Zdziarski A.A., 1989, ApJ, 342, 1108

\section{APPENDIX A: COOLING OF THE NON-THERMAL PARTICLES}

In this appendix we provide equations for the cooling rate of non-thermal electrons and protons.

The cooling rate of electrons is given by (cf. Ginzburg \& Syrovatskii 1964; Manolakou et al. 2007)

$\frac{d \gamma_{\mathrm{e}}}{d t}=b_{\mathrm{S}} \gamma_{\mathrm{e}}^{2}+b_{\mathrm{IC}} \gamma_{\mathrm{e}}^{2} F_{\mathrm{KN}}\left(\gamma_{\mathrm{e}}\right)+b_{\mathrm{C}}\left(\ln \gamma_{\mathrm{e}}+b_{\mathrm{C}}^{0}\right)+b_{\mathrm{B}} \gamma_{\mathrm{e}}\left(\ln \gamma_{\mathrm{e}}+b_{\mathrm{B}}^{0}\right)+\frac{v \gamma_{\mathrm{e}}}{R}$,

where the coefficients $b_{\mathrm{S}}, b_{\mathrm{IC}}, b_{\mathrm{C}}$ and $b_{\mathrm{B}}$ for the synchrotron, inverse Compton, coulombic and bremsstrahlung losses are given by

$b_{\mathrm{S}}=\frac{4 \sigma_{\mathrm{T}}}{3 m_{\mathrm{e}} c} U_{\mathrm{B}}=1.292 \times 10^{-15}(B / \mathrm{mG})^{2} \mathrm{~s}^{-1}$,

$b_{\mathrm{IC}}=b_{\mathrm{S}} \frac{U_{\mathrm{ph}}}{U_{\mathrm{B}}}=5.204 \times 10^{-20}\left(U_{\mathrm{ph}} / \mathrm{eV} \mathrm{cm}^{-3}\right) \mathrm{s}^{-1}$,

$b_{\mathrm{C}}=\frac{2 \pi e^{4} n_{\mathrm{e}}}{m_{\mathrm{e}}^{2} c^{3}}=1.491 \times 10^{-14} n_{\mathrm{e}} \mathrm{s}^{-1}$,

and

$b_{\mathrm{B}}=\frac{4 e^{6} n_{\mathrm{e}}}{m_{\mathrm{e}}^{2} c^{4} \hbar}=1.37 \times 10^{-16} n_{\mathrm{e}} \mathrm{s}^{-1}$.

The constants $b_{\mathrm{C}}^{0}$ and $b_{\mathrm{B}}^{0}$ are given by

$b_{\mathrm{C}}^{0}=\ln \left(\frac{m_{\mathrm{e}}^{3} c^{4}}{4 e^{2} n_{\mathrm{e}} \hbar^{2}}\right)+\frac{3}{4}=-\ln n_{\mathrm{e}}+73.4$,

and

$b_{\mathrm{B}}^{0}=\ln 2-\frac{1}{3}=0.36$.

In these equations, $\sigma_{\mathrm{T}}$ is the Thomson cross section, $U_{\mathrm{ph}}$ and $U_{\mathrm{B}}$ are the photon and magnetic field energy densities, respectively, $n_{\mathrm{e}}$ is the electron number density, while $c, m_{\mathrm{e}}$ and $e$ are the speed of light, and the electron mass and charge. For a black-body distribution of target photons,

$F_{\mathrm{KN}}\left(\gamma_{\mathrm{e}}\right) \approx\left(1+4 \gamma_{\mathrm{e}} \epsilon_{\mathrm{eff}}\right)^{-3 / 2}$,

where

$\epsilon_{\mathrm{eff}}=2.8 \frac{k T}{m_{\mathrm{e}} c^{2}}$.

This approximation takes into account the full Klein-Nishina cross-section for Compton scattering, and is valid for an anisotropic target photon field provided the electron distribution is isotropic (see Manolakou et al. 2007, and references therein).

The last term in Eq. A1 is due to the adiabatic cooling. Here $v$ is the flow speed along the contact discontinuity and $R$ is the distance of the gas from its star. We assume that the hot plasma expands almost spherically as it flows out of the system, consistent with the approach taken by del Palacio et al. (2016). 
In addition to cooling the non-thermal particles, adiabatic expansion also reduces their number density. We assume that a change in volume occurs when the non-thermal particles flow from one segment to the next, and that this change is related to the difference in the immediate postshock density of the thermal plasma between the segments. Specifically, we assume that $\rho_{1} V_{1}=\rho_{2} V_{2}$, where $\rho_{1(2)}$ and $V_{1(2)}$ are the density of the thermal particles and volume of the non-thermal particles in segment $1(2)$. The change in volume, $d V=V_{2}-V_{1}$. Thus $d V / V_{2}=\left(\rho_{1} / \rho_{2}-1\right)$.

The change of the electron Lorentz factor with time in Eq. A1 is defined to be positive for electron cooling, so

$\dot{E}_{\mathrm{e}}=-m_{\mathrm{e}} c^{2} \frac{d \gamma_{\mathrm{e}}}{d t}$.

The cooling rate of non-thermal protons is given by

$\frac{d \gamma_{\mathrm{p}}}{d t}=c n_{\mathrm{p}} \gamma_{\mathrm{p}} K_{\mathrm{pp}} \sigma_{\mathrm{pp}}\left(\gamma_{\mathrm{p}}\right)+\frac{v \gamma_{\mathrm{p}}}{R}$,

where $n_{\mathrm{p}}$ is the number density of thermal protons, $\sigma_{\mathrm{pp}}$ is the total inelastic cross section and $K_{\mathrm{pp}} \approx 0.5$ is the total inelasticity of the interaction. $\sigma_{\mathrm{pp}}$ can be approximated as (Kelner et al. 2006)

$\sigma_{\mathrm{pp}}\left(E_{\mathrm{p}}\right)=\left(34.3+1.88 L+0.25 L^{2}\right)\left[1-\left(\frac{E_{\mathrm{th}}}{E_{\mathrm{p}}}\right)^{4}\right]^{2} \mathrm{mb}$,

where $E_{\mathrm{th}}=1.22 \mathrm{GeV}$ is the threshold energy for the production of a single $\pi^{0}$.

\section{APPENDIX B: EMISSIVITIES}

In this appendix we provide equations for the emissivity calculations in our models (see Cerutti (2007) and Vila (2012) for further details).

\section{B1 Anisotropic inverse Compton emission}

Consider a target photon scattering off an electron that is moving with velocity $v=\beta c$. One can define two reference frames: $\mathrm{K}$ is the observer (lab) frame and $\mathrm{K}^{\prime}$ is the rest frame of the electron. In the lab frame the angle between the incident photon and the electron is $\theta_{0}$, and the photon energy is $\epsilon_{0}$. All the quantities defined in $K$ that are measured in K' are written with a "prime". Thus in the rest frame of the electron, the angle and energy of the incident photon are $\theta_{0}^{\prime}$ and $\epsilon_{0}^{\prime}$, respectively. The scattered photon moves at an angle $\theta_{1}$ from the direction vector of the electron in the lab frame, and at an angle of $\theta_{1}^{\prime}$ in the electron rest frame. The scattered photon has an energy $\epsilon_{1}$ in the lab frame, and an energy $\epsilon_{1}^{\prime}$ in the electron rest frame. In the lab frame the incident photon has an azimuthal angle $\phi_{0}$, while the scattered photon has an azimuthal angle $\phi_{1}$. These angles are respectively $\phi_{0}^{\prime}$ and $\phi_{1}^{\prime}$ in the electron rest frame. The geometry and parameters are shown in Fig. B1. The derivation below closely follows the work in Cerutti (2007).

The Compton formula gives

$\epsilon_{1}^{\prime}=\frac{\epsilon_{0}^{\prime}}{1+\frac{\epsilon_{0}^{\prime}}{m_{\mathrm{e}} c^{2}}\left(1-\cos \Theta^{\prime}\right)}$, where the scattering angle $\Theta^{\prime}$ is a function of the other angles of the problem:

$\cos \Theta^{\prime}=\cos \theta_{1}^{\prime} \cos \theta_{0}^{\prime}+\sin \theta_{1}^{\prime} \sin \theta_{0}^{\prime} \cos \left(\phi_{1}^{\prime}-\phi_{0}^{\prime}\right)$.

The differential cross section per solid angle $d \Omega_{1}^{\prime}$ and per energy $\epsilon_{1}^{\prime}$ of the Compton scattering for unpolarized radiation is given by the Klein-Nishina formula (see, e.g., Heitler 1954; Rybicki \& Lightman 1979)

$$
\begin{aligned}
\frac{d \sigma}{d \epsilon_{1}^{\prime} d \Omega_{1}^{\prime}}=\frac{r_{\mathrm{e}}^{2}}{2}\left(\frac{\epsilon_{1}^{\prime}}{\epsilon_{0}^{\prime}}\right)^{2}\left(\frac{\epsilon_{1}^{\prime}}{\epsilon_{0}^{\prime}}+\frac{\epsilon_{0}^{\prime}}{\epsilon_{1}^{\prime}}-\sin ^{2} \Theta^{\prime}\right) \times \\
\quad \delta\left(\epsilon_{1}^{\prime}-\frac{\epsilon_{0}^{\prime}}{1+\frac{\epsilon_{0}^{\prime}}{m_{\mathrm{e}} c^{2}}\left(1-\cos \Theta^{\prime}\right)}\right),
\end{aligned}
$$

where $r_{\mathrm{e}}$ is the classical electron radius.

Now consider a monoenergetic and unidimensional photon distribution interacting with a single energetic electron of energy $E_{\mathrm{e}}=\gamma m_{\mathrm{e}} c^{2}$. In the observer's (lab) frame this distribution (in units of photons $/ \mathrm{cm}^{3} / \mathrm{erg} / \mathrm{sr}$ ) can be written as

$n_{\mathrm{ph}}=\frac{d n}{d \epsilon d \Omega}=n_{0} \delta\left(\epsilon-\epsilon_{0}\right) \delta\left(\theta-\theta_{0}\right) \delta\left(\phi-\phi_{0}\right)$,

where $\epsilon$ is the energy of the incident photons, and $\theta$ and $\phi$ are the polar and azimuthal angle (see Figure 3.1 in Cerutti 2007). The polar axis $x$ is parallel to the initial electron momentum, so that the polar angle $\theta_{0}$ is also the collision angle.

Since $\frac{d n}{d \epsilon}$ is a Lorentz invariant, $\frac{d n}{d \epsilon}=\frac{d n^{\prime}}{d \epsilon^{\prime}}$, so

$\frac{d n^{\prime}}{d \epsilon^{\prime} d \Omega^{\prime}}=\frac{d n}{d \epsilon d \Omega} \frac{d \Omega}{d \Omega^{\prime}}$.

Using the Doppler shift formulae, Eq. B4 becomes

$$
\begin{array}{r}
\frac{d n}{d \epsilon d \Omega}=n_{0} \delta\left(\epsilon^{\prime} \gamma\left(1+\beta \cos \theta^{\prime}\right)-\epsilon_{0}\right) \delta\left(\frac{\cos \theta^{\prime}+\beta}{1+\beta \cos \theta^{\prime}}-\cos \theta_{0}\right) \times \\
\delta\left(\phi^{\prime}-\phi_{0}^{\prime}\right), \quad(\mathrm{B} 6)
\end{array}
$$

and

$\frac{d \Omega}{d \Omega^{\prime}}=\gamma^{2}(1-\beta \cos \theta)^{2}=\frac{1}{\gamma^{2}\left(1+\beta \cos \theta^{\prime}\right)^{2}}$.

The Dirac distribution has the property that for a function $f(x)$ where for all $i, f\left(x_{i}\right)=0$, then

$\delta(f(x))=\sum_{i} \frac{1}{|d f / d x|_{x=x_{i}}} \delta\left(x-x_{i}\right)$.

It is then possible to express the differential photon density in $\mathrm{K}^{\prime}$ as

$$
\begin{aligned}
\frac{d n^{\prime}}{d \epsilon^{\prime} d \Omega^{\prime}}=n_{0} \gamma\left(1-\beta \cos \theta_{0}\right) \delta\left(\epsilon^{\prime}-\epsilon_{0} \gamma\left(1-\beta \cos \theta_{0}\right)\right) \times & \\
& \delta\left(\cos \theta^{\prime}-\frac{\cos \theta_{0}-\beta}{1-\beta \cos \theta_{0}}\right) \delta\left(\phi^{\prime}-\phi_{0}^{\prime}\right) .
\end{aligned}
$$

To obtain the inverse Compton spectrum per electron, we first need to determine the differential number of scattered photons (photons $/ \mathrm{s} / \mathrm{sr}^{2} / \mathrm{erg}^{2}$ ) in the rest frame of the electron, which is

$\frac{d N}{d t^{\prime} d \epsilon_{1}^{\prime} d \Omega_{1}^{\prime} d \epsilon^{\prime} d \Omega^{\prime}}=\frac{d n^{\prime}}{d \epsilon^{\prime} d \Omega^{\prime}} c \frac{d \sigma}{d \epsilon_{1}^{\prime} d \Omega_{1}^{\prime}}$.

However, the observer is interested in the differential number 

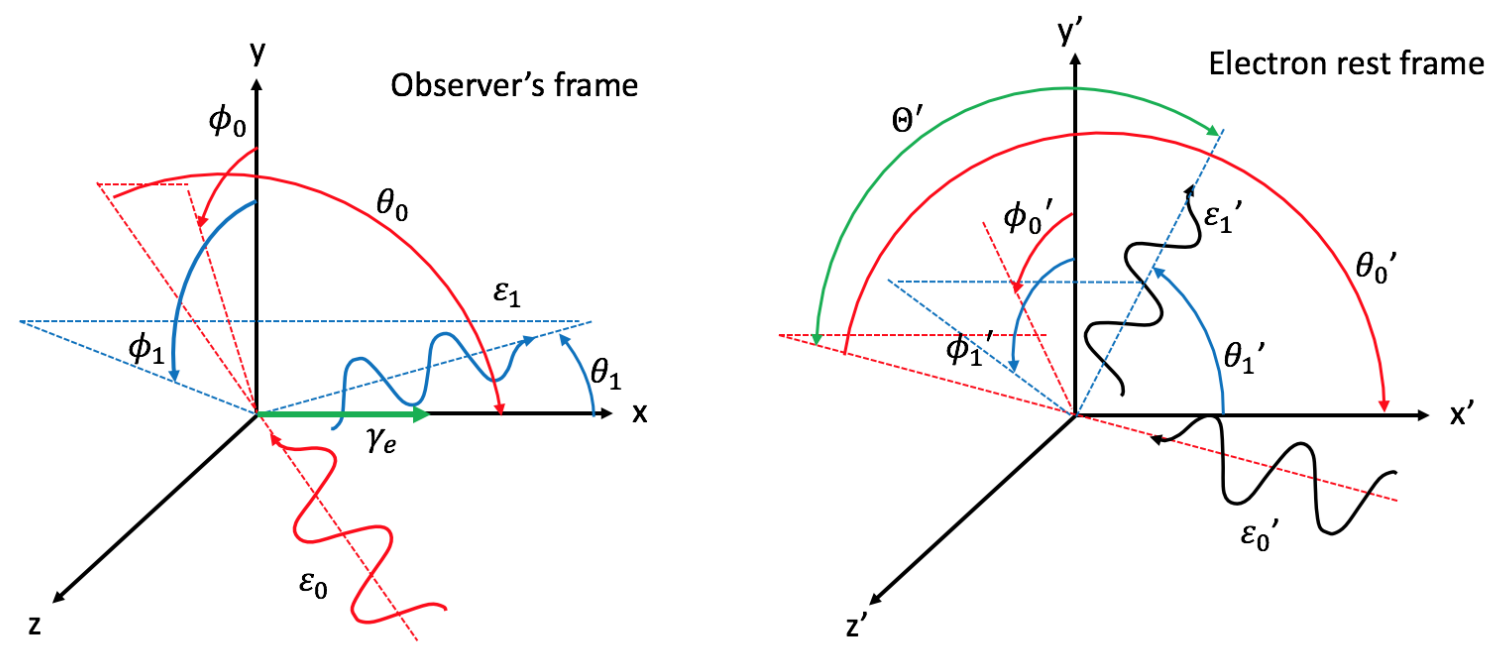

Figure B1. The inverse Compton scattering geometry and parameters in the observer's frame (left) and the rest frame of the electron (right). The incident and scattered photons are represented by waves and the green arrow shows the direction of motion of the electron in the lab frame. The Lorentz boost from the observer to the rest frame of the electron is along the $x$-axis.

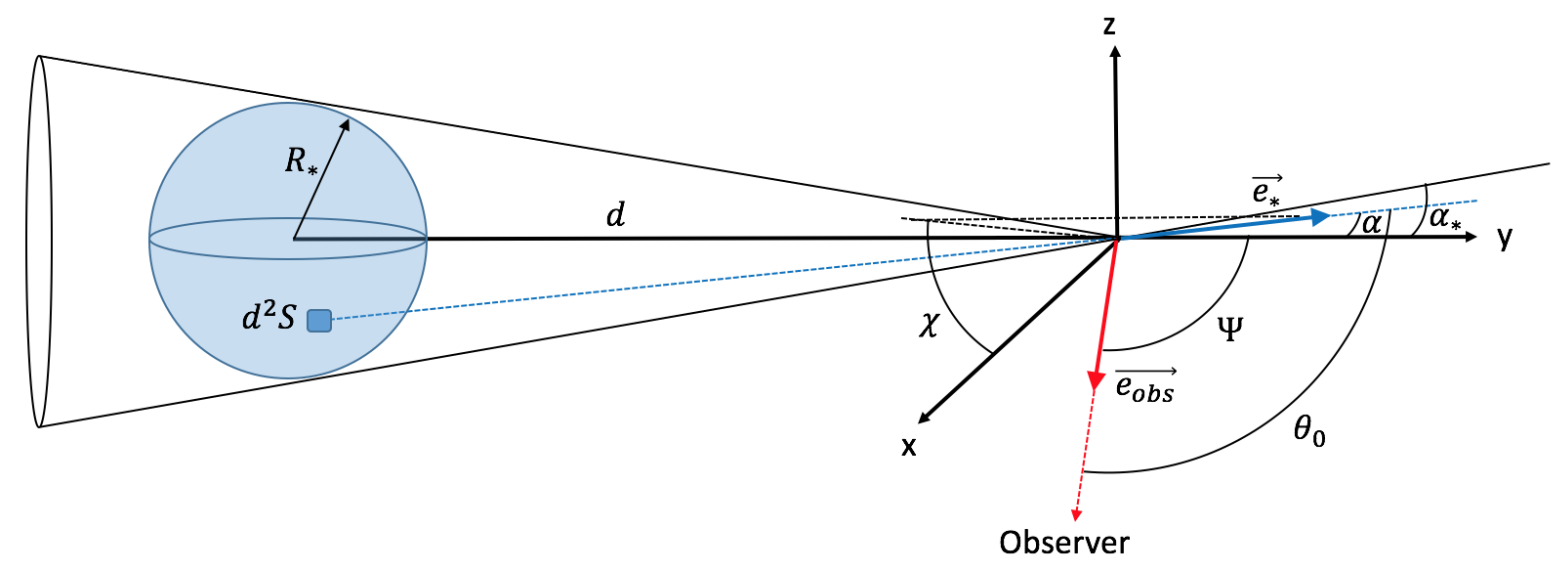

Figure B2. The geometry of the star-electron-observer. The blue arrow shows the direction of the incoming stellar photon, and the red arrow shows the direction of the up-scattered photon. Because the inverse Compton emission from energetic electrons is highly beamed this is also the direction vector of the electron. The aperture angle of the star is $\alpha_{*}$, the viewing angle is $\psi$, and the scattering angle is $\theta_{0}$. The $y$-axis is defined to be the polar axis. $\chi$ is the azimuthal angle in the $x z$-plane, and $\psi$ is in the $x y$-plane.

of scattered photons per electron, per unit of time, per unit of energy $\epsilon_{1}$ and per unit of solid angle $\Omega_{1}$ in the lab frame, which is given by

$\frac{d N}{d t d \epsilon_{1} d \Omega_{1}}=\int_{\Omega^{\prime}} \int_{\epsilon^{\prime}} \frac{d N}{d t^{\prime} d \epsilon_{1}^{\prime} d \Omega_{1}^{\prime} d \epsilon^{\prime} d \Omega^{\prime}} \frac{d t^{\prime}}{d t} \frac{d \Omega_{1}^{\prime}}{d \Omega_{1}} \frac{\epsilon_{1}^{\prime}}{\epsilon_{1}} d \Omega^{\prime} d \epsilon^{\prime}$.

The Jacobian of the Lorentz transformation from $\mathrm{K}^{\prime}$ to $\mathrm{K}$ is

$\frac{d t^{\prime}}{d t} \frac{d \Omega_{1}^{\prime}}{d \Omega_{1}} \frac{\epsilon_{1}^{\prime}}{\epsilon_{1}}=\frac{1}{\gamma} \frac{1}{\gamma^{2}\left(1-\beta \cos \theta_{1}\right)^{2}} \gamma\left(1-\beta \cos \theta_{1}\right)$.

Thus one obtains

$\frac{d N}{d t d \epsilon_{1} d \Omega_{1}}=\frac{1}{\gamma^{2}\left(1-\beta \cos \theta_{1}\right)} \int_{\Omega^{\prime}} \int_{\epsilon^{\prime}} \frac{d n^{\prime}}{d \epsilon^{\prime} d \Omega^{\prime}} c \frac{d \sigma}{d \epsilon_{1}^{\prime} d \Omega_{1}^{\prime}} d \Omega^{\prime} d \epsilon^{\prime}$.
Using the Dirac distribution property (Eq. B8), and defining $\mu=\cos \Theta^{\prime}$, Eq. B3 becomes

$$
\delta\left(\epsilon_{1}^{\prime}-\frac{\epsilon^{\prime}}{1+\frac{\epsilon^{\prime}}{m_{\mathrm{e}} c^{2}}(1-\mu)}\right)=
$$

$$
\frac{1}{\left[1-\frac{\epsilon_{1}^{\prime}}{m_{\mathrm{e}} c^{2}}(1-\mu)\right]^{2}} \delta\left(\epsilon^{\prime}-\frac{\epsilon_{1}^{\prime}}{1-\frac{\epsilon_{1}^{\prime}}{m_{\mathrm{e}} c^{2}}(1-\mu)}\right) \text {. }
$$


Eq. B13 then becomes

$$
\begin{aligned}
& \frac{d N}{d t d \epsilon_{1} d \Omega_{1}}=\frac{1}{\gamma^{2}\left(1-\beta \cos \theta_{1}\right)} \int_{\Omega^{\prime}} \int_{\epsilon^{\prime}} n_{0} \gamma\left(1-\beta \cos \theta_{0}\right) \times \\
& \delta\left(\epsilon^{\prime}-\epsilon_{0} \gamma\left(1-\beta \cos \theta_{0}\right)\right) \delta\left(\cos \theta^{\prime}-\cos \theta_{0}^{\prime}\right) \delta\left(\phi^{\prime}-\phi_{0}^{\prime}\right) \times \\
& \frac{r_{\mathrm{e}}^{2}}{2}\left(\frac{\epsilon_{1}^{\prime}}{\epsilon^{\prime}}\right)^{2}\left(\frac{\epsilon_{1}^{\prime}}{\epsilon^{\prime}}+\frac{\epsilon^{\prime}}{\epsilon_{1}^{\prime}}-1+\mu^{2}\right) \frac{1}{\left[1-\frac{\epsilon_{1}^{\prime}}{m_{\mathrm{e}} c^{2}}(1-\mu)\right]^{2}} \times \\
& \delta\left(\epsilon^{\prime}-\frac{\epsilon_{1}^{\prime}}{1-\frac{\epsilon_{1}^{\prime}}{m_{\mathrm{e}} c^{2}}(1-\mu)}\right) d \epsilon^{\prime} d \Omega^{\prime} .
\end{aligned}
$$

These integrations are straightforward and give

$$
\begin{aligned}
\frac{d N}{d t d \epsilon_{1} d \Omega_{1}}= & \frac{r_{\mathrm{e}}^{2} n_{0} c\left(1-\beta \cos \theta_{0}\right)}{2 \gamma\left(1-\beta \cos \theta_{1}\right)} \times \\
& {\left[1+\mu^{2}+\left(\frac{\gamma \epsilon_{1}}{m_{\mathrm{e}} c^{2}}\right)^{2} \frac{\left(1-\beta \cos \theta_{1}\right)^{2}(1-\mu)^{2}}{1-\frac{\gamma \epsilon_{1}}{m_{\mathrm{e}} c^{2}}\left(1-\beta \cos \theta_{1}\right)(1-\mu)}\right] \times } \\
& \delta\left(\frac{\gamma \epsilon_{1}\left(1-\beta \cos \theta_{1}\right)}{1-\frac{\gamma \epsilon_{1}}{m_{\mathrm{e}} c^{2}}\left(1-\beta \cos \theta_{1}\right)(1-\mu)}-\gamma \epsilon_{0}\left(1-\beta \cos \theta_{0}\right)\right) .
\end{aligned}
$$

The integration over $\Omega_{1}$ is complicated, but can be obtained by making use of the approximation that for $\gamma>>1$,

$\mu \approx \frac{\cos \theta_{1}-\beta}{1-\beta \cos \theta_{1}} C_{\theta_{0}}$,

where

$C_{\theta_{0}}=\frac{\cos \theta_{0}-\beta}{1-\beta \cos \theta_{0}}$.

Because of this approximation, the spectrum is independent of the azimuthal angle and the integration over the azimuthal angle $\phi_{1}$ just multiplies it by $2 \pi$. The remaining integration over $x=\cos \theta_{1}$ is simple as well. If $\mu_{0}=\mu\left(x_{0}\right)$, the number of photons with final energy $\epsilon_{1}$ scattered into all outward directions per unit time (photons/s/erg) is then

$$
\begin{aligned}
\frac{d N}{d t d \epsilon_{1}}\left(E_{\mathrm{e}}, \epsilon_{0}, \epsilon_{1}\right) & =\pi r_{\mathrm{e}}^{2} n_{0} c K \frac{\left(1-\beta \cos \theta_{0}\right)}{\gamma\left(1-\beta x_{0}\right)} \times \\
& {\left[1+\mu_{0}^{2}+\left(\frac{\gamma \epsilon_{1}}{m_{\mathrm{e}} c^{2}}\right)^{2} \frac{\left(1-\beta x_{0}\right)^{2}\left(1-\mu_{0}\right)^{2}}{1-\frac{\gamma \epsilon_{1}}{m_{\mathrm{e}} c^{2}}\left(1-\beta x_{0}\right)\left(1-\mu_{0}\right)}\right], }
\end{aligned}
$$

where

$x_{0}=\frac{1-\frac{\epsilon_{0}}{\epsilon_{1}}\left(1-\beta \cos \theta_{0}\right)+\frac{\gamma \epsilon_{0}}{m_{\mathrm{e}} c^{2}}\left(1-\beta \cos \theta_{0}\right)\left(1+\beta C_{\theta_{0}}\right)}{\beta+\frac{\gamma \epsilon_{0}}{m_{\mathrm{e}} c^{2}}\left(1-\beta \cos \theta_{0}\right)\left(\beta+C_{\theta_{0}}\right)}$,

and

$K=\frac{\left\{1-\frac{\gamma \epsilon_{1}}{m_{\mathrm{e}} c^{2}}\left[1+\beta C_{\theta_{0}}-\left(\beta+C_{\theta_{0}}\right) x_{0}\right]\right\}^{2}}{\left|-\beta \gamma \epsilon_{1}-\frac{\epsilon_{1}^{2}}{m_{\mathrm{e}} c^{2}} C_{\theta_{0}}\right|}$

Eq. B19 is only valid between the energy limits $\epsilon_{\min } \leq \epsilon_{1} \leq$ $\epsilon_{\max }$, where

$\epsilon_{\min / \max }=\frac{\gamma m_{\mathrm{e}} c^{2}\left(1-\beta \cos \theta_{0}\right) \epsilon_{0}}{\gamma m_{\mathrm{e}} c^{2}+\epsilon_{0} \pm \sqrt{\epsilon_{0}^{2}+m_{\mathrm{e}}^{2} c^{4} \gamma^{2} \beta^{2}+2 \epsilon_{0} \beta \gamma m_{\mathrm{e}} c^{2} \cos \theta_{0}}}$.

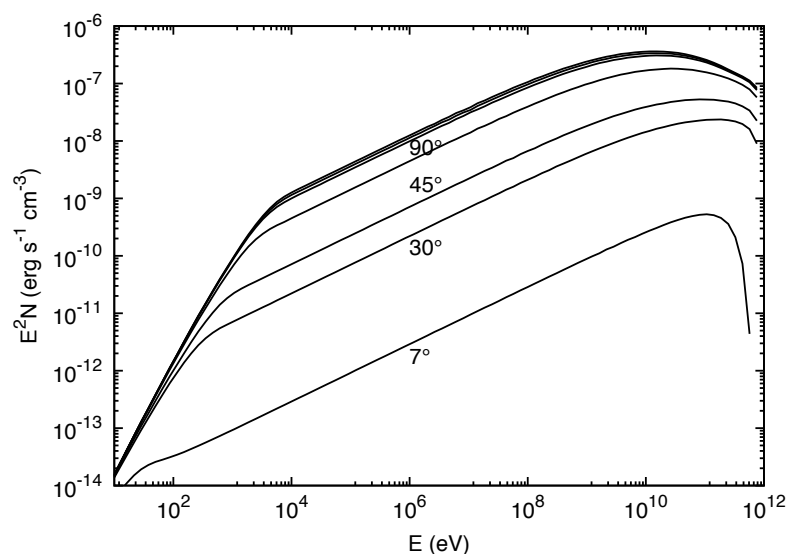

Figure B3. The inverse Compton luminosity $L_{\gamma}=E_{\gamma}^{2} N_{\gamma}\left(E_{\gamma}\right)$ calculated for a point-like star and different values of the viewing angle $\psi$. The energy distribution of the incident photons is a blackbody with $T=3.9 \times 10^{4} \mathrm{~K}$. The electron distribution is a power-law $N_{\mathrm{e}}\left(E_{\mathrm{e}}\right) \propto E_{\mathrm{e}}^{-2}$ over the energy range $10 m_{\mathrm{e}} c^{2} \leq E_{\mathrm{e}} \leq 1 \mathrm{TeV}$.

To obtain the total emission, Eq. B19 must be integrated over the incident photon and electron distributions, the collision angle, and the volume containing the nonthermal particles, $V$. For incident photons from a star, the geometry is illustrated in Fig. B2, which is based on Fig. 4.1 in Cerutti (2007). The polar axis $y$ is chosen so that it joins the centre of the star and the interaction site, which are separated by a distance $d$. The direction vector for any photon emitted by the star can be written as

$\vec{e}_{*}=(\sin \alpha \cos \chi, \cos \alpha, \sin \alpha \sin \chi)$

$\chi$ can take the range $0 \leq \chi \leq 2 \pi$, but the polar angle is limited due to the size of the star to the range $0 \leq \alpha \leq \alpha_{*}$, where $\alpha_{*}=\arcsin \left(R_{*} / d\right)$ and $R_{*}$ is the stellar radius.

If the system is seen with a viewing angle $\psi$, then the scattered photon has the unit vector

$\vec{e}_{\mathrm{obs}}=(\sin \psi, \cos \psi, 0)$.

Because the inverse Compton emission from energetic electrons is highly beamed this is also the direction vector of the electron, $\vec{e}_{e}$. The collision angle, $\theta_{0}$, can then be obtained from the scalar product of $\vec{e}_{e}$ and $\vec{e}_{*}$ :

$\vec{e}_{e} \cdot \vec{e}_{*}=\cos \theta_{0}=\cos \psi \cos \alpha+\sin \psi \sin \alpha \cos \chi$.

The resulting emission (photons/s/erg) is given by

$$
\begin{aligned}
& \frac{d N}{d t d \epsilon_{1}}=\int_{V} d V \int_{E_{\mathrm{e}, \min }}^{E_{\mathrm{e}, \max }} \int_{\epsilon_{0, \min }}^{\epsilon_{0, \max }} \int_{0}^{2 \pi} \int_{0}^{\alpha_{*}} \frac{d N}{d t d \epsilon_{1}}\left(E_{\mathrm{e}}, \epsilon_{0}, \alpha, \chi\right) \times \\
& n_{\mathrm{ph}}\left(\epsilon_{0}\right) N_{\mathrm{e}}\left(E_{\mathrm{e}}\right) \cos \alpha \sin \alpha d \alpha d \chi d \epsilon_{0} d E_{\mathrm{e}},
\end{aligned}
$$

where $n_{\mathrm{ph}}\left(\epsilon_{0}\right)$ is the number density of incident photons at energy $\epsilon_{0}$ (in units of photons $/ \mathrm{cm}^{3} / \mathrm{erg} / \mathrm{sr}$ ), $N_{\mathrm{e}}\left(E_{\mathrm{e}}\right)$ is the nonthermal electron distribution (in units of electrons $/ \mathrm{erg} / \mathrm{cm}^{3}$ ), and $\cos \alpha \sin \alpha d \alpha d \chi$ is the projection of an element of area $d^{2} S$ on the surface of the star.

In Fig. B3 the variarion of the inverse Compton emissivity with viewing angle is shown for a point-like star with a blackbody photon distribution scattering off non-thermal electrons with a power-law energy distribution. The effect of the anisotropy is clear to see. Most of the variation is 
between $0^{\circ}<\psi<90^{\circ}$, and there is virtually no change for $135^{\circ}<\psi<180^{\circ}$.

\section{B2 Relativistic bremsstrahlung emission}

The $\gamma$-ray emission (photons/s/erg) from relativistic bremsstrahlung resulting from the interaction of nonthermal electrons with thermal protons is (e.g. Blumenthal \& Gould 1970)

$q_{\gamma}\left(E_{\gamma}\right)=c \int_{V} d V n_{\mathrm{p}} \int_{E_{\mathrm{e}}^{\min }}^{E_{\mathrm{e}}^{\max }} \frac{d \sigma_{\mathrm{Br}}}{d E_{\gamma}}\left(E_{\gamma}, E_{\mathrm{e}}\right) N_{\mathrm{e}}\left(E_{\mathrm{e}}\right) d E_{e}$,

where $E_{\gamma}$ is the photon energy, $n_{\mathrm{p}}$ is the number density of thermal protons, $E_{\mathrm{e}}$ is the energy of the non-thermal electron, and $E_{\mathrm{e}}^{\max }$ and $E_{\mathrm{e}}^{\min }$ are the maximum and minimum energy of the non-thermal electrons. The differential cross section (in units of $\mathrm{cm}^{2} / \mathrm{erg}$ ) for the emission of a photon by a non-thermal electron (with energy $E_{\mathrm{e}}>>m_{\mathrm{e}} c^{2}$ ) in the presence of a proton is (e.g., Berezinskii et al. 1990)

$$
\begin{aligned}
\frac{d \sigma_{\mathrm{Br}}}{d E_{\gamma}}\left(E_{\gamma}, E_{e}\right)=\frac{4 \alpha_{\mathrm{FS}} r_{\mathrm{e}}^{2}}{E_{\gamma}}[1+ & \left.\left(1-\frac{E_{\gamma}}{E_{e}}\right)^{2}-\frac{2}{3}\left(1-\frac{E_{\gamma}}{E_{e}}\right)\right] \times \\
& \left\{\ln \left[\frac{2 E_{e}\left(E_{e}-E_{\gamma}\right)}{m_{\mathrm{e}} c^{2} E_{\gamma}}\right]-\frac{1}{2}\right\},
\end{aligned}
$$

where $\alpha_{\mathrm{FS}}$ is the fine structure constant.

\section{B3 $\pi^{0}$-decay emission}

The $\gamma$-ray emission (photons/s/erg) from the decay of neutral pions is

$q_{\gamma}\left(E_{\gamma}\right)=2 \int_{E_{\min }}^{E_{\mathrm{p}}^{\max }} \frac{Q_{\pi^{0}}\left(E_{\pi}\right)}{\sqrt{E_{\pi}^{2}-m_{\pi^{0}}^{2} c^{4}}} d E_{\pi}$,

where $E_{\pi}$ is the neutral pion energy and

$$
E_{\min }=E_{\gamma}+\frac{m_{\pi^{0}}^{2} c^{4}}{4 E_{\gamma}} .
$$

$Q_{\pi^{0}}$ is the injection function of neutral pions (pions/s/erg). In the delta functional approximation it is given by (Aharonian \& Atoyan 2000)

$Q_{\pi^{0}}\left(E_{\pi}\right)=\int_{V} d V \frac{\tilde{n}}{K_{\pi}} c n_{\mathrm{p}} \sigma_{\mathrm{pp}}\left(E_{\mathrm{p}}\right) N_{\mathrm{p}}\left(E_{\mathrm{p}}\right)$,

where $\tilde{n}$ is the number of neutral pions created per protonproton collision (it is assumed that $\tilde{n}=1$ and does not depend on the energy of the proton), $K_{\pi}=0.17$ is the fraction of the proton kinetic energy that goes into creating the pion, $n_{\mathrm{p}}$ is the number density of thermal protons, and $N_{\mathrm{p}}\left(E_{\mathrm{p}}\right)$ is the non-thermal proton distribution (in units of protons $/ \mathrm{erg} / \mathrm{cm}^{3}$ ) where the proton energy is $E_{\mathrm{p}}$. The inelastic proton-proton cross-section, $\sigma_{\mathrm{pp}}$, is accurately approximated as (Kelner, Aharonian \& Bugayov 2006)

$\sigma_{\mathrm{pp}}\left(E_{\mathrm{p}}\right)=\left(34.3+1.88 L+0.25 L^{2}\right)\left[1-\left(\frac{E_{\mathrm{th}}}{E_{p}}\right)^{4}\right]^{2} \mathrm{mb}$,

where $L=\ln \left(E_{p} / 1 \mathrm{TeV}\right)$ and $E_{\mathrm{th}}=\left(m_{p}+2 m_{\pi}+m_{\pi}^{2} / 2 m_{p}\right) c^{2}=$ $1.22 \mathrm{GeV}$ is the threshold energy for the production of a single $\pi^{0}$.
This paper has been typeset from a $\mathrm{T}_{\mathrm{EX}} / \mathrm{LAT} \mathrm{T} \mathrm{X}$ file prepared by the author. 